\title{
Genes and Pathways Induced in Early Response to Defoliation in Rice Seedlings
}

\author{
Shuangyan Chen ${ }^{1}$, Xiu-Qing $\mathrm{Li}^{2}$, Aiguo Zhao ${ }^{1,4}$, \\ Lijuan Wang ${ }^{1,4}$, Xiaofeng $\mathrm{Li}^{1,4}$, Qingyun $\mathrm{Sh}^{3}$, \\ Ming Chen ${ }^{3}$, Juan Guo ${ }^{1,4}$, Jichong Zhang ${ }^{1,4}$, \\ Dongmei $\mathrm{Qi}^{1}$, and Gongshe Liü*
}

${ }^{1}$ Institute of Botany, Chinese Academy of Sciences, 100093
Beijing, P. R. China
${ }^{2}$ Molecular Genetics Laboratory, Potato Research Centre,
Agriculture and Agri-Food Canada, 850 Lincoln Road, P.O.
Box 20280 , Fredericton, NB, E3B $4 Z 7$, Canada
${ }^{3}$ The National Key Laboratory of Plant Physiology and
Biochemistry, College of Life Sciences, Zhejiang University,
Hangzhou 310058, China
${ }^{4}$ Graduate School of the Chinese Academy of Sciences,
100093 Beijing, P. R. China

\begin{abstract}
How plant gene expression respond to grazing defoliation is critical for plant re-growth, survival, and composition in the natural and dairy farming grassland environments. Rice, with genome sequence available, was used as a model plant to study grazing-induced pathway selections. When seedlings were $18 \mathrm{~cm}$ in height, the top $12 \mathrm{~cm}$ was removed by simulated grazing. The gene expression activities within 2 to 24 hours in the remaining aboveground tissues were profiled using the Affymetrix Rice GeneChips and RTqPCR. The seedlings responded to grazing by immediately adjusting their global gene expression, e.g. enhancing anaerobic respiration, starch-to-sugar conversion, sucrose synthesis, and sugar transport. The results suggest that 1) remaining aboveground tissues used anaerobic respiration as an emergency measure for energy/substrates supply; 2) Sink tissues reduced its demand after $2 h ; 3$ ) Sucrose synthesis enhancement around the $24^{\text {th }}$ hour is likely driven by shoot re-growth. In total, the expression activity of 466 genes, involved in signal transduction, miRNA regulation, cell wall modification, metabolism, hormone synthesis, and molecule transporters, had been significantly changed at least twofold. These genes and their biochemical pathways identified provide insights into how plants respond to grazing at the molecular physiology level.
\end{abstract}

\section{Introduction}

Grassland grass defoliation can be caused mainly by grazing, although by icy-storms and insects at certain degrees. Considerable variation in plant composition and sustainability of natural or dairy-farming grasslands can be due to grazing defoliation by wild or dairy animals such as cows (Brueland et al., 2003), sheep (Jauregui et al., 2007), deer (Vourch' et al., 2002), rabbits (Crawley, 1990), and kangaroos (Meers and Adams, 2003). Without grazing, grasslands would not be

*Corresponding author: Email: liugs@ibcas.ac.cn the same as we see now. Grazing influences the abundance, distribution, and persistence of plant species in environment with recurrent grazing defoliation (Donaghy and Fulkerson, 2002; Pecetti and Piano, 2002; Del-Val and Crawley, 2005). For example, rabbit grazing increased the abundance of Anthoxanthum odoratum and the Rumex acetosella grasses and decreased the abundance of Festuca rubra and Agrostis capillaris grasses (Crawley, 1990). Grazing is a multiple-component process that includes wounding, saliva depositing, and defoliation, etc. The sustainability of various plant species to grazing has likely resulted from the adaptation in various physiological and stress-response pathways. Although grass photosynthesis, secondary metabolism, carbohydrates relocation, and re-growth after grazing have been documented (Detling et al., 1979; Nowak and Caldwell, 1984; Gold and Caldwell, 1990; Orodho and Trlica, 1990; Belesky and Hill, 1997; McPherson and Williams, 1998), it is unclear how rapidly and through which biochemical pathways the seedlings achieve the observed growth-level response.

Among the biochemical pathways usually involved in the response to stresses in plants, anaerobic respiration under anoxia is known to play an important role in the generation of both cellular energy and biosynthesis-required substrates (Givan, 1999). Flooding and gas limitation are among the most known anoxic stresses (Kato-Noguchi et al., 2003; Agarwal and Grover, 2006). Anaerobic respiration is required during anoxia but not yet found for other environmental stresses, although anaerobic respiration is suspected to play an important role in non-anoxic tissues (Kursteiner et al., 2003). In the present study, we hypothesized that anaerobic respirations may be activated as an emergencyrescue approach even in leaf tissues in the air when grasses are grazed.

Recent breakthroughs in molecular biology have provided scientists with exciting new tools to pursue eco-genomics research. Microarray technique has been successfully applied to ecology studies (Gibson, 2002) and has identified genes that may be involved in plant-insect interactions (Reymond et al., 2000; Moran et al., 2002; Reymond et al., 2004; Voelckel et al., 2004). After Arabidopsis plants were infested with two types of caterpillars (Pieris rapae and $P$. brassicae), the plants showed highly similar gene expression profiles (Reymond et al., 2000; Reymond et al., 2004). In Arabidopsis plants, gene expression profile changes induced by caterpillar and thrips showed highest degrees of overlap, followed by thrips and aphid induced changes. However, caterpillar and aphid induced changes hardly overlapped at all (De Vos et al., 2005). Although gene expression profiling has proved to be very informative in studying Arabidopsis-herbivore interaction, to the best of our knowledge, there is no report that evaluates the gene expression profile of the early response in grasses to grazing. Because of the critical importance of gene activities for grass re-growth and survival after grazing, this study aimed to identify the candidate genes and their biochemical 
pathways using rice, the model monocot plant, mainly because its whole genome has been sequenced (Goff et al., 2002).

\section{Materials and methods}

\section{Plant materials}

Rice (Oryza sativa sp. japonica) cultivar Zhonghua No. 10 was grown in a greenhouse with a $12 \mathrm{~h}$ light cycle, at $29^{\circ} \mathrm{C}$ during the day and $21^{\circ} \mathrm{C}$ during the night. The relative humidity was approximately from $50 \%$ to $70 \%$. Germinated rice seeds were grown in a plastic container $(\mathrm{LxWxH}$ : $9 \times 9 \times 10 \mathrm{~cm}$ ) filled with rice nursery culture soil (containing $0.15 \mathrm{~g}$ each of nitrogen, phosphate, and potassium). Twelve rice seedlings were planted in each plastic container. When the seedlings were at approximately $18 \mathrm{~cm}$ in height (about 3-4 leaves per seedling), the top $12 \mathrm{~cm}$ part including all the full-size leaf blades was removed by simulated grazing with cow saliva daubing using cotton stick at cut surface immediately after clipping. The shoot meristem was not damaged. The inside main meristem remained unhurt. The remaining aboveground parts (nearly no leaf blades) were collected 2, 6, and $24 \mathrm{~h}$ after grazing (Figure 1). For the control seedlings (unclipped), the corresponding parts in ungrazed seedlings were collected. Microarray (2 h) hybridizations and/or RT-qPCR (2, 6 and $24 \mathrm{~h})$ experiments were separately for each of the three independent biological replicates. Each sample was created from pooling at least 20 rice seedlings from two plastic containers. The samples were immediately frozen in liquid nitrogen and stored at $-80^{\circ}$ for RNA analysis.

RNA isolation, cRNA synthesis, and hybridization to Affymetrix Rice GeneChips

Total RNA was extracted with Trizol reagent (Invitrogen, Carlsbad, CA, USA) and NucleoSpin $\AA$ RNA Clean-up (CapitalBio company, China). RNA quality was assessed by agarose gel electrophoresis and spectrophotometry. RNA was processed for use on the 51,279-gene Affymetrix Rice Genome GeneChip arrays (Affymetrix, Inc, Santa Clara, CA, USA), according to the manufacturer's protocol. In brief, $10 \mathrm{mg}$ of total RNA was used in the reverse transcription reaction (Ambion MessageAmp kit) to generate the firststrand cDNA. After the second-strand synthesis, the doublestrand CDNA was transcribed in vitro to generate biotinylated cRNA. After purification and fragmentation, the biotinylated cRNA was used for hybridization. The hybridization, washing, staining, and scanning procedures were performed by CapitalBio company (Beijing, China), as described in the Affymetrix technical manual. The biotin-labelled targets were hybridized to the GeneChip Rice Genome Array for $16 \mathrm{~h}$ at $45^{\circ} \mathrm{C}$ with rotation at 60 r.p.m. in an Affymetrix GeneChip Hybridization Oven 640. Washing and staining were carried out in an Affymetrix Fluidics Station 450, following the protocol for the standard format of antibody amplification for eukaryotic targets (EuKGEWS2v4). The processed arrays were scanned with an Agilent GeneArray Scanner (Agilent, Palo Alto, CA, USA).

\section{Data analysis}

The hybridization data were analyzed using GeneChip Operating Software (GCOS 1.4) and dChip software (Li and Wong, 2001, 2001) (http://www.dchip.org). The scanned images were examined for any visible defects. Satisfactory images were analyzed to generate raw data saved as CEL files using the default settings of GCOS 1.4 from Affymetrix. A global scaling factor of 500 , a normalization value of 1 , and default parameter settings for the rice genome array were used. The detection calls (present, absent, or marginal) for the probe sets were made by GCOS. Further analysis was done using dChip. The normalization of all arrays was performed using an invariant set approach. For calculating the expression index of probe sets, we used the perfect match/mismatch model and setup "absent" by truncating the low-expression values to the tenth percentile of the probe set signals. The expression values were $\log _{2}$ transformed

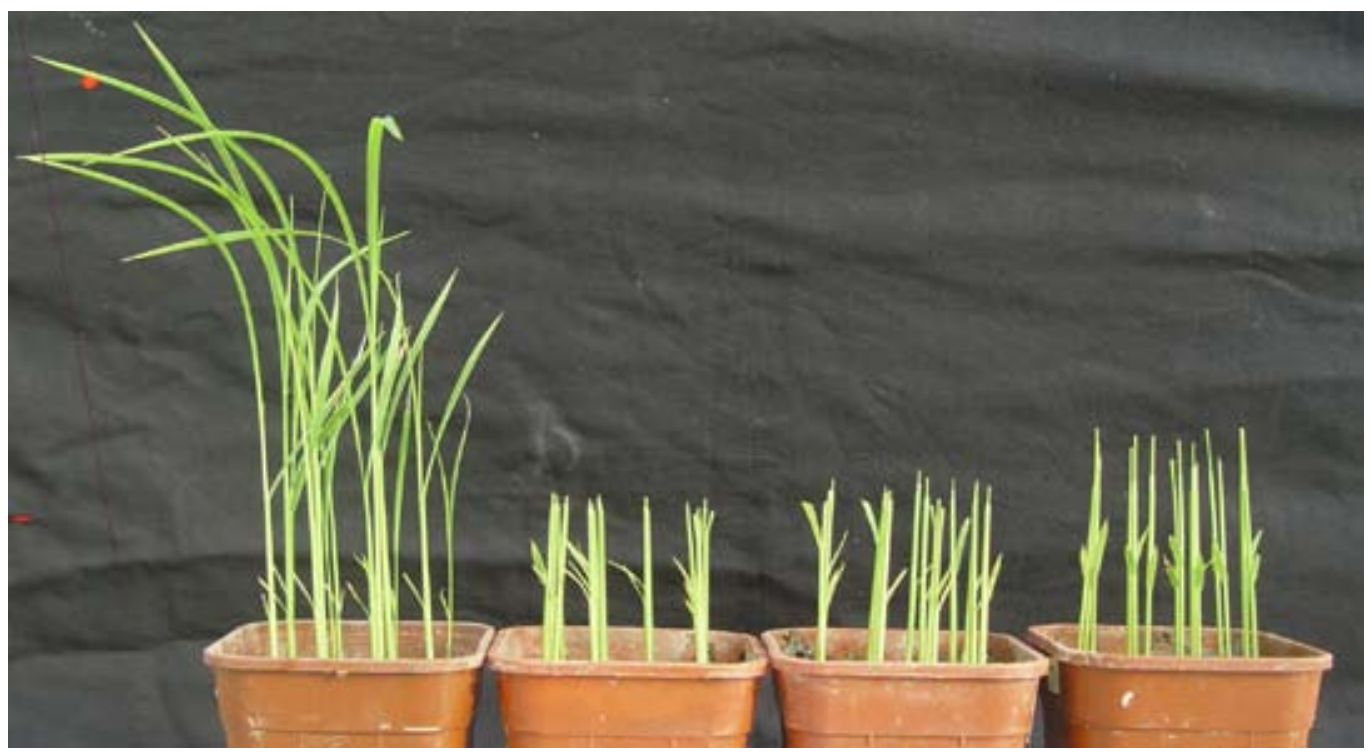

Figure 1. Rice seedlings at 2, 6, and $24 \mathrm{~h}$ after simulated grazing. From left to right: the control plants and the grazing plants at 2,6 , and $24 \mathrm{~h}$, respectively. 


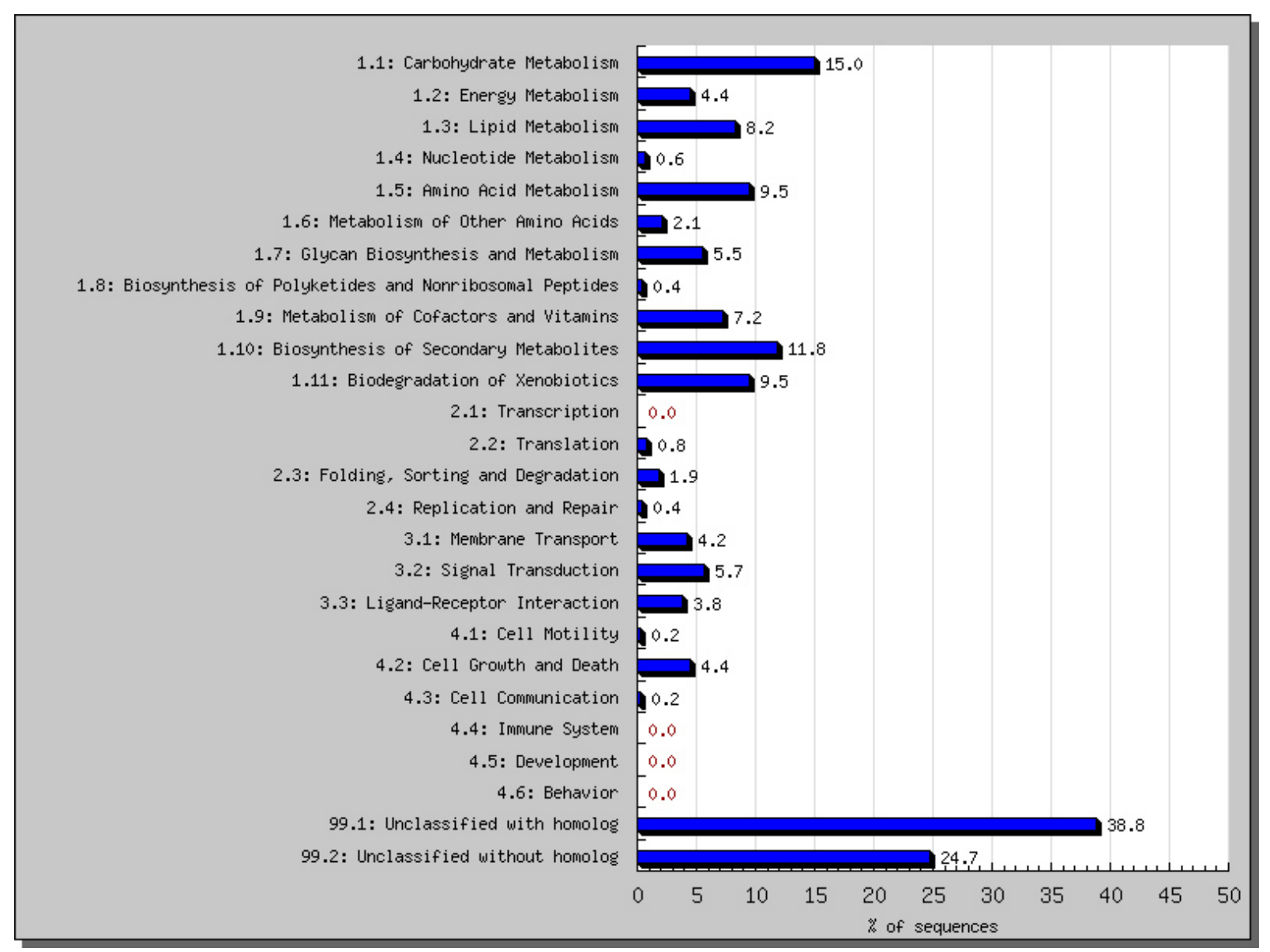

Figure 2. Functional distribution in the GeneBins ontology analysis of differential expression probe sets at $2 \mathrm{~h}$. The percentage represents the proportion of submitted probe sets that have been assigned in the corresponding categories (BIN).

after calculating the expression index. The signal log ratio is the change in the expression level of a transcript between the control and experimental samples, and $\log _{2}$ (ratio) $\geq 1$ (2-fold change) or $\log _{2}$ (ratio) $\leq-1$ ( 0.5 -fold change) was used as cutoff. The Significance Analysis of Microarrays software package (Tusher et al., 2001) was utilized to analyze the three repeated samples between grazing and control using $q$ value $\leq 0.05$ and fold-change $\geq 2$ as cutoff.

We used GeneBins to define a generic hierarchical classification. GeneBins can be used to identify the functional categories associated with a set of sequences (e.g. differentially expressed) and thus find the metabolic pathways or other cellular functions up- or down-regulated in microarray experiments (Goffard and Weiller, 2007). The sum of the percentages can be more than $100 \%$ as a gene can be assigned to several BINs.

\section{RT-qPCR}

The total RNA was treated with DNase I to avoid DNA contamination. One microgram of RNA was reverse transcribed using the Superscript II reverse transcriptase (Invitrogen) with an oligo d(T18) primer according to manufacturer's instructions. PCR experiments were conducted using RealMasterMix (SYBR Green) (Tiangen Biotech, Beijing, China). Reactions were carried out on a Rotor-Gene 3000 multicolor real-time detection system. The following standard thermal profile was used for all
PCR experiments: $95^{\circ} \mathrm{C}$ for $10 \mathrm{~min} ; 45$ cycles of $95^{\circ} \mathrm{C}$ for $15 \mathrm{~s}, 55^{\circ} \mathrm{C}$ for $20 \mathrm{~s}$ and $68{ }^{\circ} \mathrm{C}$ for $20 \mathrm{~s}$; then $72{ }^{\circ} \mathrm{C}$ for $10 \mathrm{~min}$. Fluorescence signals were captured at the end of each cycle, and the melting curve analysis was performed from $72{ }^{\circ} \mathrm{C}$ to $95^{\circ} \mathrm{C}$. Gene-specific primers were designed using the Primer3 software (Table S1). Data analysis was performed with the Rotor-Gene 6 software. The relative quantification method was used to evaluate quantitative variation between replicates examined. The amplification of actin gene sequence (Os03g0718100) was used as the internal control to normalize all the data.

\section{Results}

\section{Gene expression profile in grazing-treated rice seedlings}

Within 24 hours after clipping/grazing, the young leaves at the centre of the seedling shoot re-grew to approximately $3 \mathrm{~cm}$ longer (Figure 1). When RNA from the aboveground parts harvested $2 \mathrm{~h}$ after clipping was used in hybridization analysis against the 51,279 genes on the GeneChip arrays, the expression activity of 466 probe sets changed at least 2 fold ( $q \leq 0.05$ ) in the grazed seedlings, compared to the corresponding ungrazed seedlings (Table S2).

In the generic hierarchical classification of the 466 genes (Figure 2), the main classes were carbohydrate metabolism $(15 \%)$, biosynthesis of secondary metabolism $(11.8 \%)$, 
A

Probe sets ID Os.27606.1.S1_at Os.27650.1. $1^{-a t}$ Os.55671.1.51 Os.9216.1.s1_ât Os.1310.1.s1-a_a Os.18607.1.51_at Os.15426.1.S1_at Os.10555.1.S1_a_at Os.51063.1.51 at Os.46800.1.52 -a_at Os.50342.1.81_a Os.12012.1.S1_at Os.53502.1.51-at Os.7028.1.s1_at Os.37565.1.51 $\mathrm{x}$ s.12032.1.s1_at s.7339.1.s1_at S.54232.1.S1_at S.52616.1.A1_at S.51959.1.82_at s.36152.1.51 at s.38278.1.51 -at S.32634.1.51_at S.54944.1.81_at s.48082.1.51 at s.10356.1.81_at s.30670.1.51_at S.54934.1.81_at Os.26695.1.S1_at S.27092.1.S1_at s.31858.1.S1_at s.55259.1.s1_at s.15138.1.81_at S.37996.1.s1 at . s.25606.11296.1.s1_at at s.4804.1.s1 at s.10635.1.51 s at Os.8696.1.51_x at s.7902.1.81 at s.14882.1.51_at s.19553.1. S1s.9554.1.s1 at

B

Putative function

spartate kinase family protein

rotein kinase domain containing protein Protein kinase domain containing protein

Paemolysin-ll related family protein

omain containing protein C.interacting

Receptor-like protein kinase homolog RK20-1

Zinc finger, $\mathrm{C} 2 \mathrm{H} 2$ type family protein

F-box family protein

Lelix-loop-helix DNA-binding domain containing protein Leucine Rich Repeat family protein

Leucine-rich repeat receptor protein kinase EXS precursor (M)

列 政

MRK DNA binding domain containing protein

WRK $Y$ transcription factor

$\mathrm{CHY}$ zinc finger family protein

Zinc finger, $\mathrm{C} 2 \mathrm{H} 2$ type family protein

Serine/threonine-protein kinase receptor precursor

Zinc finger, $\mathrm{C} 2 \mathrm{H} 2$ type family protein

B3 DNA binding domain containing protein

Myb-like DNA-binding domain containing protein

2 domain containing protein

RKY DNA binding domain containing protein

Zliu motif family protein

Myb-like DNA-binding domain containing protein

NAC-domain containing protein 19

Protein kinase domain containing protein

Receptor-like serine-threonine protein kinase

ZlM motif family prote

MCB2 protein

EF hand family protein, expressed

aining protein

AP2 domain containing protein

GHMP kinases putative ATP-binding protein

IQ calmodulin-binding motif family protein

EF-hand $\mathrm{Ca} 2+$-binding protein $\mathrm{CCD}$

WRKY DNA binding domain containing protein

G-box binding factor 1
Probe sets ID Os.8266.1.A1_at Os.405.1.s1 a -at Os.27112.1.51 $\overline{\mathrm{at}}^{\mathrm{at}}$ Os.8778.1.51 $\bar{a}^{\mathrm{a}}$ at Os.51641.1.S1_at S. $553861 . \mathrm{Al}^{-a t}$ Os.11608.1.51-at os.19952.1.51_at s.53660.1. $51^{-}$ Os.31778.1.51-x at os.17449.1.A1 -at Os.17356.1.A1_A Os.11812.1.S1_at

D

Putative

Os. 4179.1 .51 at Os.47946.1.sर $\mathrm{s}$ _at

os.28427.1.s2_a_at

Os. 4166.1 .81 at

Os.9212.1.81_at

s.7314.1.51-at

Os.49091.1.s1_at

Os.28032.1.A1_at

OS.12391.1.S1

Os.6776.1.s1_at

Os.4159.1.81_at

os.12952.1.s1_at

Os.32115.1.s1_at

Os.10215.1.s1-at

Os.10908.1.s1_a_at

Os.313.1.s1_a_at

0 . $36264.1 . \overline{5} 1$ at

os.19003.1.s1_a_at

Os.21893.3.A1_a

Os.27755.1.81_at

Os.54829.1.s1-at

Os.51106.1.81_at

Putative function

tochrome P450 74A

SAM dependent carboxyl methyltransferase family protein ipoxygenase 2.

(2)

2-oxophytodienoate reductase 2

-aminocyclopropane-1-carboxylate oxidase

- Aundent carboxy methyltranserase family protein ene responsive protein

enescence-associated protein DIN

Auxin efflux carrier comnonent 6
Function

4-alpha-glucan branching enzyme

Glucose-6-phosphateiphosphate translocator 2

\section{Polvgalacturonase}

Glucose-1-phosphate adenyly|transferase

Granule-bound starch synthase $1 \mathrm{~b}$

Glucan 1,3-beta-glucosidase

Sucrose phosphate synthase

Endoglucanase

Glucan endo-1,3-beta-glucosidase 5

Trehalose-6-phosphate synthase

Glucan endo-1,3-beta-glucosidase 3

Glucan endo-1,3-beta-glucosidase Gll

Glucan endo-13-beta-glucosidase Gl

Glucan endo-1,3-beta-glucosidase Gll

$L$-lactate dehydrogenase A

Alpha-amylase isozyme 3D

Aldehyde dehydrogenase

Glucan endo-1,3-beta-glucosidase 4

Pyruvate dehydrogenase

Trehalose-6-phosphate synthase

Glucan endo-1,3-beta-D-glucosidase

Endoglucanase 1

Endoglucanase 1

sudar transporter family protein
C

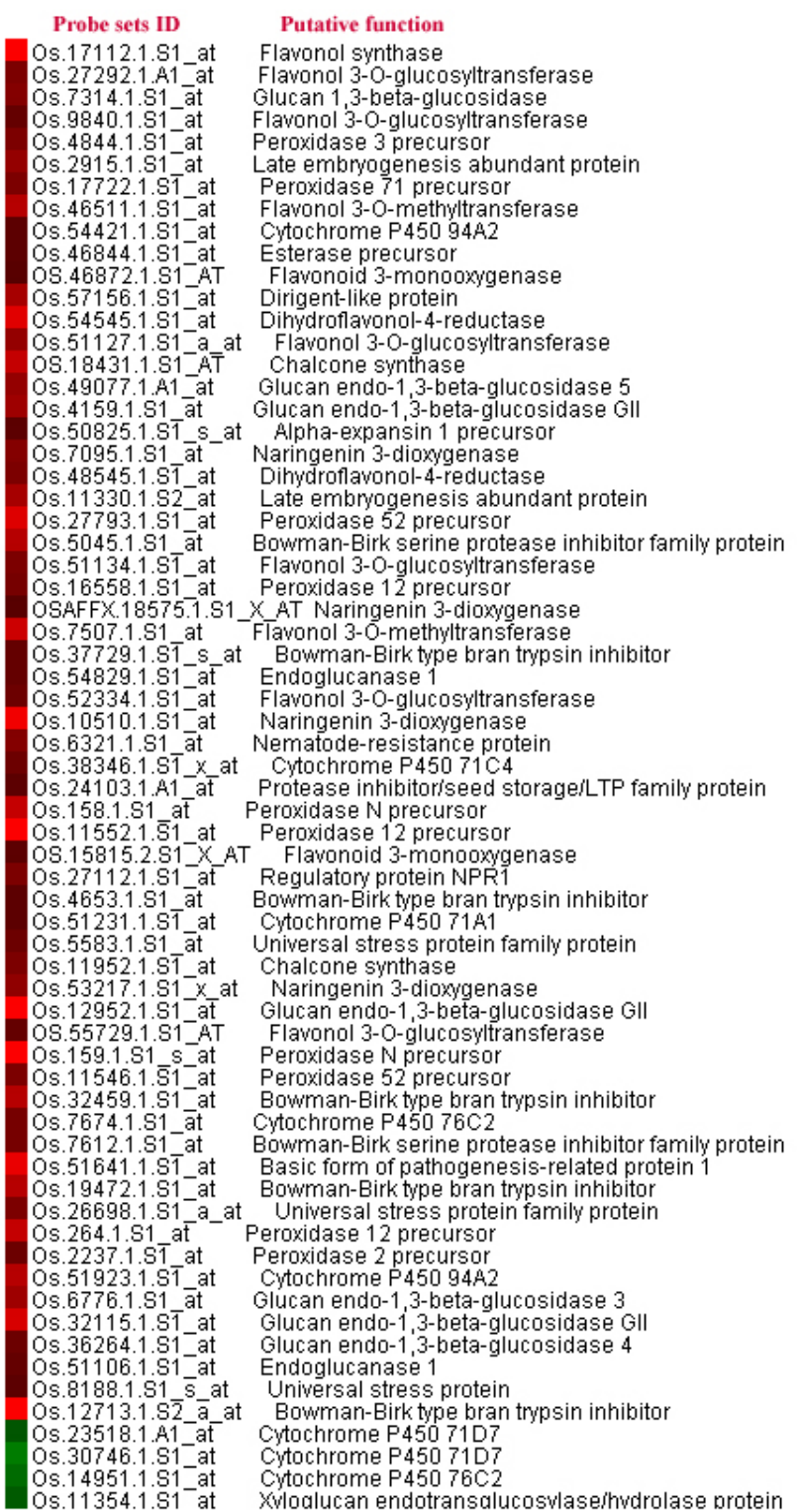

Figure 3. Four major functional groups of genes with at least twofold activity changes at $2 \mathrm{~h}$ according to the microarray analysis. A (Group I): Genes involved in signal transduction and transcription factors; B (Group II): Hormonal signaling pathways genes; C (Group III): Secondary metabolism and defense-related genes; and D (Group IV): Carbohydrate metabolism genes. Green to bright red: down regulation to up-regulation. 
Table 1. Description of 30 grazing responsive genes used in RT-qPCR analysis.

\begin{tabular}{|c|c|c|c|c|c|c|}
\hline \multirow[t]{2}{*}{ Probe set ID / Gene product } & \multirow[t]{2}{*}{ Metabolism pathways } & \multirow[t]{2}{*}{ Figure 3 group } & \multicolumn{4}{|c|}{ Activity changes (fold, $\log 2$ transformed) } \\
\hline & & & Microarray $2 \mathrm{~h}$ & RT-qPCR 2h & RT-qPCR $6 \mathrm{~h}$ & RT-qPCR 2 \\
\hline $\begin{array}{l}\text { Os.25606.1.S1_at / WRKY DNA binding domain } \\
\text { containing protein }\end{array}$ & Transcription factor & Group I & 2.87 & 2.66 & 1.48 & 0.70 \\
\hline $\begin{array}{l}\text { Os.54934.1.S1_at / myb-like DNA-binding domain } \\
\text { containing protein }\end{array}$ & Transcription factor & Group I & 4.08 & 4.32 & 3.67 & 3.04 \\
\hline $\begin{array}{c}\text { Os.27759.1.S1_at / Protein kinase domain } \\
\text { containing protein }\end{array}$ & Signal transduction & Group I & -1.94 & -2.32 & -2.40 & -1.60 \\
\hline $\begin{array}{l}\text { Os.15426.1.S1_at / Receptor-like protein kinase } \\
\text { homolog RK20-1 }\end{array}$ & Signal transduction & Group I & 2.03 & 1.58 & 1.30 & 1.45 \\
\hline $\begin{array}{l}\text { Os.19617.1.S1_at / Leucine Rich Repeat family } \\
\text { protein }\end{array}$ & Signal transduction & Group I & 1.55 & 1.87 & 0.66 & -0.36 \\
\hline $\begin{array}{l}\text { Os.19952.1.S1_at / OsIAA2 - Auxin-responsive } \\
\text { Aux/IAA gene family member }\end{array}$ & Hormone signaling & Group II & 2.44 & 2.06 & 2.22 & 2.41 \\
\hline $\begin{array}{l}\text { Os.11608.1.S1_at / OsIAA6 - Auxin-responsive } \\
\text { Aux/IAA gene family member }\end{array}$ & Hormone signaling & Group II & 1.41 & 1.24 & 0.90 & 1.10 \\
\hline Os.17449.1.A1_at / Auxin efflux carrier component 6 & Hormone signaling & Group II & -1.18 & -1.29 & -0.40 & 0.11 \\
\hline Os.405.1.S1_a_at / Lipoxygenase 2.1 & Hormone signaling & Group II & 2.64 & 2.27 & 2.24 & 0.40 \\
\hline Os.27112.1.S1_at / Regulatory protein NPR1 & Hormone signaling & Group II & 1.50 & 1.40 & 1.13 & 1.10 \\
\hline Os.54545.1.S1_at / dihydroflavonol-4-reductase & Flavonoid biosynthesis & Group III & 2.67 & 2.12 & 0.79 & -0.53 \\
\hline Os.17112.1.S1_at / flavonol synthase & Flavonoid biosynthesis & Group III & 2.95 & 3.60 & 2.15 & 1.06 \\
\hline Os.10510.1.S1_at/naringenin 3-dioxygenase & Flavonoid biosynthesis & Group III & 2.87 & 2.65 & 1.87 & 0.46 \\
\hline Os.46511.1.S1_at / flavonol 3-O-methyltransferase & Flavonoid biosynthesis & Group III & 2.14 & 2.56 & 0.89 & 0.56 \\
\hline Os.18431.1.S1_at/chalcone synthase & Flavonoid biosynthesis & Group III & 2.31 & 2.27 & 0.20 & 1.02 \\
\hline Os.51127.1.S1_a_at / flavonol 3-O-glucosyltransferase & Flavonoid biosynthesis & Group III & 1.72 & 1.48 & 0.92 & 0.61 \\
\hline Os.7314.1.S1_at / glucan 1,3-beta-glucosidase & Starch and sucrose metabolism & Group IV & 1.53 & 2.31 & 1.37 & 1.16 \\
\hline Os.4179.1.S1_at / 1,4-alpha-glucan branching enzyme & Starch and sucrose metabolism & Group IV & -1.03 & -1.06 & -1.25 & -1.03 \\
\hline $\begin{array}{l}\text { Os.47946.1.S1_s_at / glucose-6-phosphate/phosphate } \\
\text { translocator } 2\end{array}$ & Starch and sucrose metabolism & Group IV & -1.43 & -1.64 & -1.79 & -1.60 \\
\hline Os.49091.1.S1_at / sucrose phosphate synthase & Starch and sucrose metabolism & Group IV & 1.27 & 1.48 & 0.95 & 1.10 \\
\hline Os.21893.3.A1_at / trehalose-6-phosphate synthase & Starch and sucrose metabolism & Group IV & 2.45 & 2.23 & 2.49 & 2.55 \\
\hline Os.9212.1.S1_at / granule-bound starch synthase 1b & Starch and sucrose metabolism & & -1.51 & -2.94 & -3.84 & -3.47 \\
\hline $\begin{array}{l}\text { Os.4166.1.S1_at / glucose-1-phosphate } \\
\text { adenylyltransferase }\end{array}$ & Starch and sucrose metabolism & Group IV & -1.89 & -1.89 & -3.18 & -2.84 \\
\hline Os.313.1.S1_a_at / aldehyde dehydrogenase & Pyruvate metabolism & Group IV & 1.73 & 1.53 & 1.71 & 1.90 \\
\hline Os.21906.1.S1_at / sugar transporter family protein & Transport & Group IV & 1.16 & 1.01 & 0.69 & 0.51 \\
\hline
\end{tabular}

amino acid metabolism (9.5\%), and lipid metabolism $(8.2 \%)$. Genes classified to membrane transport, signal transduction, and ligand-receptor interaction were $4.2 \%$, $5.7 \%$ and $3.8 \%$, respectively. Genes involved in cell growth and death was $4.4 \%$.

The activity changes for four functional gene groups including signal transduction and transcription factors, hormonal signaling pathways, secondary metabolism and defense, and carbohydrate metabolism genes are demonstrated in Figure 3.

The validation of microarray results was conducted using RT-qPCR analysis of 30 differentially expressed genes covering all the four functional groups and both upregulated and down-regulated genes (Table 1). These genes were chosen, based on biochemistry knowledge, from those involved in flavonoid biosynthetic pathways, carbohydrate metabolism, hormone signaling, signal transduction and transcription factors. The expression activities determined by RT-qPCR data correlate strongly with the changes identified by the microarray experiments $\left(R^{2}=0.93\right.$, Figure S1).

\section{Signal transduction and transcription factors}

Several receptor-like kinase genes in the signal transduction pathways were activated (Figure $3 \mathrm{~A}$ ), including genes encoding receptor-like serine-threonine protein kinase, and receptor-like protein kinase homolog RK20-1. A number of non-receptor protein kinase genes were also induced, including serine/threonine-protein kinases, GHMP kinases putative ATP-binding protein, calmodulin-binding protein, leucine rich repeat family protein, and cyclin delta-2. Expression changes were also observed in several transcription factors such as AP2, WRKY, MYB, Helixloop-helix, NAC, EF hand, F-box motif, bHLH, and bZIP families.

\section{Hormonal signaling pathways}

A cytochrome P450 74A1 gene, a lipoxygenase 2.1 gene, a 12-oxophytodienoate reductase gene, and two SAM dependent carboxyl methyltransferase family protein genes were up-regulated by grazing (Figure 3B). Each of these genes are involved in jasmonic acid (JA) synthesis. The genes up-regulated by grazing include also an ethylene synthesis gene encoding 1-aminocyclopropane-1carboxylate oxidase 1 (ACO 1), two ethylene-responsive protein genes, two AUX/IAA family protein genes, and a gibberellin-20 oxidase-2 gene (Figure 3B). 


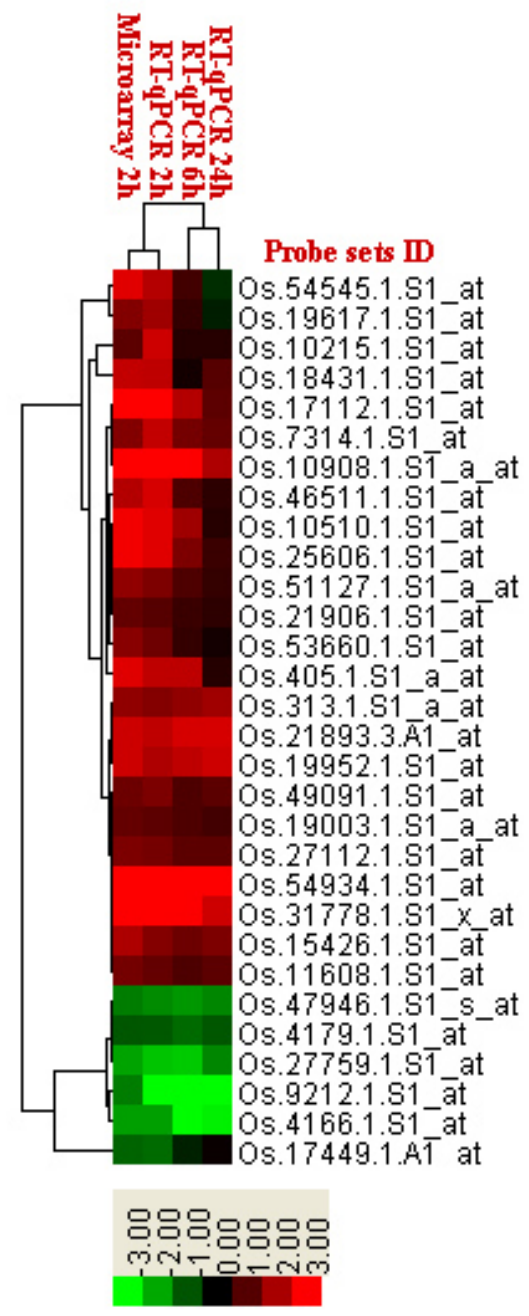

Putative function

dihydroflavonol-4-reductase

Leucine Rich Repeat family protein

$\mathrm{L}$-lactate dehydrogenase $\mathrm{A}$

chalcone synthase

flavonol synthase

glucan 1,3-beta-glucosidase

alpha-amylase isozyme

flavonol 3-0-methyltransferase

naringenin 3-dioxygenase

WRKY DNA binding domain containing protein

flavonol 3-0-glucosyltransferase

sugar transporter family protein

Ethylene responsive protein

Lipoxygenase 2.1

aldehyde dehydrogenase

trehalose-6-phosphate synthase

OSIAA2 - Auxin-responsive Aux|AA gene family member

sucrose phosphate synthase

pyruvate dehydrogenase

Regulatory protein NPR1

myb-like DNA-binding domain containing protein

Gibberellin 20 oxidase 2

Receptor-like protein kinase homolog RK20-1

OsIAA6 - Auxin-responsive AuX|AA gene family member

glucose-6-phosphateiphosphate translocator 2

1,4-alpha-glucan branching enzyme

Protein kinase domain containing protein

granule-bound starch synthase $1 \mathrm{~b}$

glucose-1-phosphate adenyly|transferase

Auxin efflux carrier component 6

Figure 4. Clustering analysis of the 30 selected grazing responsive genes based on the gene activities detected by microarray hybridization ( $2 \mathrm{~h})$ and RT-qPCR (2h, $6 \mathrm{~h}$ and $24 \mathrm{~h})$. Green to bright red: down regulation to up-regulation.

\section{Cell wall synthesis, secondary metabolism, and defense-related genes}

Among the genes induced by grazing (Figure 3C), there are also those involved in cell wall construction, modification, cell wall expansion, maintenance of cell wall architecture, and cellulose degradation, such as alpha-expansin, endoglucanases, and class III peroxidases. Gene expression of several fungal cell wall polysaccharides degradation enzymes such as glucan endo-1,3-beta-glucosidase genes were considerably induced.

Genes encoding several enzymes involved in the flavonoid biosynthetic pathways were significantly induced after grazing (Figure $3 \mathrm{C}$ and Figure 5), including two dihydroflavonol-4-reductase genes, one flavonol synthase genes, four naringenin 3-dioxygenase genes, two flavonoid 3-monooxygenase genes, two flavonol 3-O-methyltransferase genes, six flavonol 3-O-glucosyltransferase genes, and two chalcone synthase genes.

An esterase precursor, a protein involved in alkaloid biosynthesis, was also significantly up-regulated (Figure $3 C$ ). Several cytochrome family genes which are involved in secondary metabolite biosynthesis were also elevated (Figure 3C).

Grazing treatment enhanced the expressions of several protease inhibitor genes including Bowman-Birk type inhibitors (Figure 3C). Two late embryogenesis abundant genes (Figure $3 \mathrm{C}$ ), also known as dehydrins were also strongly induced by grazing. Several defense -related enzymes, including regulatory protein NPR1, pathogenesisrelated protein 1 , and nematode-resistance protein were also induced by grazing (Figure $3 \mathrm{C}$ ).

\section{Carbohydrate metabolism}

Within two hours after grazing, genes involved in starch synthesis were down-regulated (Figure 3D), including genes encoding glucose-1-phosphate adenylyltransferase, granule-bound starch synthase 1b, 1,4-alpha-glucan branching enzyme, and glucose-6-phosphate/phosphate translocator. The expression of alpha-amylase, which is involved in starch degradation, was strongly activated (Figure 3D). For sucrose synthesis and transportation, genes encoding sucrose phosphate synthase and sugar transporter were up-regulated (Figure 3D). Among the activated genes, 


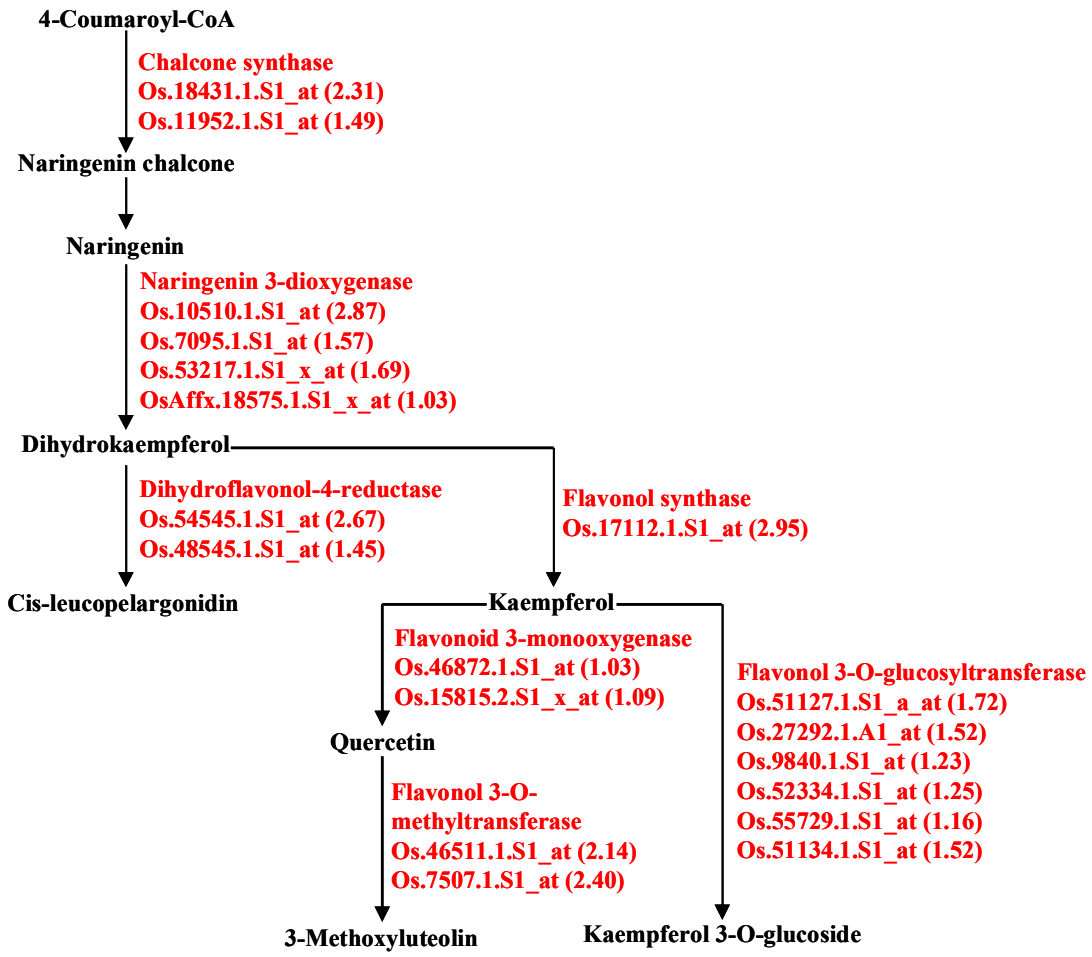

Figure 5. A simplified scheme of the flavonoid biosynthesis pathway. Enzymes encoded by genes that are induced by grazing at $2 \mathrm{~h}$ are in red. The expression difference $(\log 2)$ between the control plants and the grazed plants is listed in brackets. Note: Genes that are significantly induced in response to grazing are shown in red letters. there were also pyruvate anaerobic metabolism pathway genes (Figure 3D), including those encoding enzymes of pyruvate dehydrogenase, aldehyde dehydrogenase, and L-lactate dehydrogenase. The metabolism pathways of these differential expression genes are shown in Figure 6.

In the RT-qPCR experiments during the 24 hours after grazing (Table 1, Figures 4), the gene expression levels remained up-regulated were the following carbohydrate metabolism enzymes: alpha-amylase (Os.10908.1.S1_a_ at), aldehyde dehydrogenase (Os.313.1.S1_a_at), glucan 1,3-beta-glucosidase (Os.7314.1.S1_at), trehalose6-phosphate synthase (Os.21893.3.A1_at), pyruvate dehydrogenase (Os.19003.1.S1_a_at), and aldehyde dehydrogenase (Os.313.1.S1_a_at). The down-regulation was found for the genes encoding enzymes of glucose-1phosphate adenylyltransferase (Os.4166.1.S1_at), granulebound starch synthase 1b (Os.9212.1.S1_at), glucose-6phosphate/phosphate translocator 2 (Os.47946.1.S1_s_at), 1,4-alpha-glucan branching enzyme (Os.4179.1.S̄1_at), lactate dehydrogenase (Os.10215.1.S1_at) and sugar transporter family protein gene (Os.21906.1.S1_at).

\section{Interaction between grazing and wounding or insect feeding}

Grazing-induced genes in this study were compared to the genes induced by wounding (Lan et al., 2005) and insect feeding (Yuan et al., 2008) in rice. The genes responsive to grazing, wounding, or insect feeding were clustered and shown in Figure 7. A number of genes for metabolic enzymes, signal transduction and transcription factors, and defense-related genes were also induced by insect feeding. Some genes were induced by wounding but not by insect feeding such as peroxidase genes, cysteine synthase gene, proteasome, bZIP transcription factor, Myb-like DNA-binding domain, DnaJ domain containing protein, etc. Some genes encoding WRKY transcription factor, Leucine Rich Repeat family protein, transporters, proteinase inhibitor, hydrolase, oxidoreductase, lipoxygenase, etc. were both induced by wounding and insect feeding.

\section{Discussion}

In grasslands, since the wounding, saliva depositing, and defoliation occur during grazing as a joint process, this first study focused on the integrated effects of the simulated grazing. Effects of each separated factor of the simulated grazing will be studied in the follow-up experiments and will be reported elsewhere.

The strong stimulation of grazing immediately activates genes involved in signal transduction, cell wall metabolism, secondary metabolism, biostress defense and carbohydrate metabolism, either related to the preparation of resistance to wounding and potential pathogen attacks or the seedling regrowth. Genes responsive to grazing identified from rice may serve as candidate genes for molecular physiological studies and genetic improvement of other grass species since rice shows great synteny and high sequence similarity with other cereal genomes (Goff et al., 2002).

\section{Hormonal pathways related to regrowth or cellular functions}

Two genes encoding AUX/IAA family proteins, OsIAA2 and OsIAA6, were activated by grazing (Figure 3B). It was reported that the expression of Aux/IAA proteins represses the auxin signaling through heterodimerization with auxin response factors (ARFs) (Liscum and Reed, 2002). The binding of these transcription factors to auxin-responsive elements (AuxREs) in promoters of primary auxin-response genes may activate or repress transcription (Hagen and 


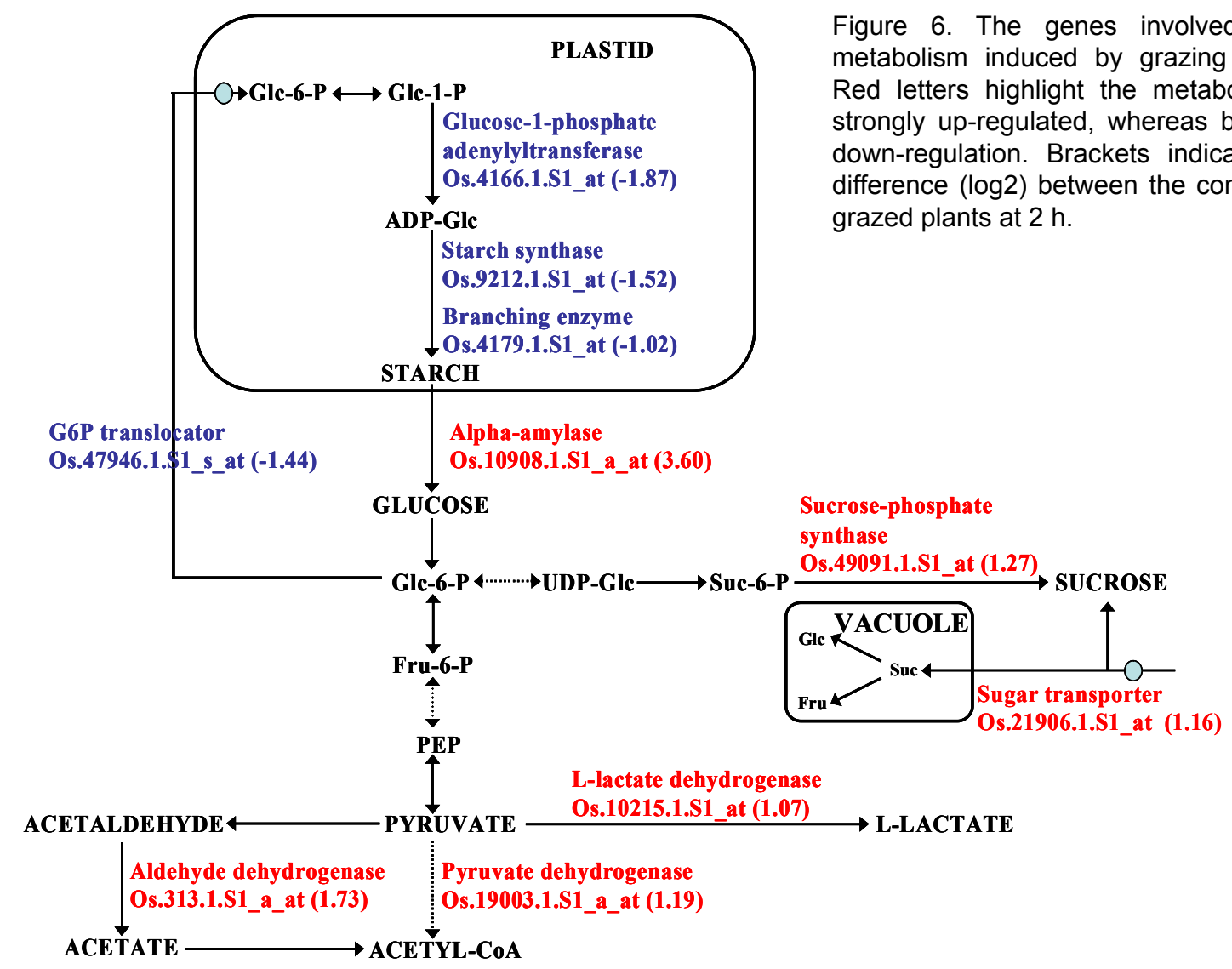

Guilfoyle, 2002). Auxin signaling in plants is involved in resistance to bacterial pathogens (Navarro et al., 2006). As part of a plant-induced immune response, bacterial pathogen-associated molecular pattern (PAMP) recognition down-regulates auxin signaling in Arabidopsis by targeting auxin receptor transcripts. These results indicate that decreasing plant auxin signaling can increase the resistance to bacterial pathogens (Navarro et al., 2006). These previous reports suggested that the up-regulated expressions of two Aux/IAA proteins (OsIAA2 and OsIAA6) might repress auxin signaling and increase the resistance to potential pathogens induced by wound under stimulated grazing. Wounding can repress auxin actions (Cheong et al., 2002) and cause IAA levels to decline (Thornburg and Li, 1991).

Ethylene participates in a variety of defense and abiotic stress responses (Ecker, 1995). In this study, the induction of genes that are involved in the ethylene biosynthetic pathway, such as 1-aminocyclopropane-1-carboxylate oxidase 1 may increase the ethylene level and subsequently activate ethylene response genes such as genes involved in senescence.

The gibberellin-20 oxidase gene up-regulated by grazing (Figure 3B) in rice seedling encodes a gibberellinsynthesis-required key enzyme that catalyzes the conversion of GA53 to GA20 via a three-step oxidation at C-20 of the GA skeleton. The upregulation of this gene suggests that grazing may increase gibberellin biosynthesis within two hours after grazing.

\section{Stress- and defense-related gene expression}

Many studies have also indicated that JA and its derivatives play an important role in the defense mechanism against different types of herbivores in Arabidopsis (McConn et al., 1997; Stintzi et al., 2001; Stotz et al., 2002; Reymond et al., 2004; Van Poecke and Dicke, 2004). Grazing-induced insect resistance cross-talks with the salicylic acid and jasmonic acid signal transduction pathways and the pathogeninduced systemic, acquired resistance (Felton et al., 1999). A regulatory protein NPR1 gene and a pathogenesis-related protein PRB1-3 gene were up-regulated in this study (Figure $3 \mathrm{C}$ ). The expression of these genes have been previously reported to be upregulated after wounding in Arabidopsis (Zhang et al., 1999). NPR1 is a critical component of the salicylic acid (SA)-mediated signal transduction pathway leading to the induction of defense genes, such as the pathogenesis-related (PR-1) gene, and the enhancement of disease resistance (Zhou et al., 2000). Pathogenesisrelated genes were also induced after wounding (Kim et al., 2005) and insects feeding (Figure 7) (Yuan et al., 2008) in rice and in Arabidopsis suspension cell cultures (Guan and Nothnagel, 2004).

Protease inhibitors are often induced by both herbivory and wounding. They are defense-related proteins that occur naturally in a wide range of plants and are considered to be an essential part in the plant direct defense against herbivores. A Bowman-Birk type protease inhibitor and a seed storage protease inhibitor responsive to grazing were also wounding-induced proteins (Figure 7). Most of protease inhibitors identified in this study were also induced 


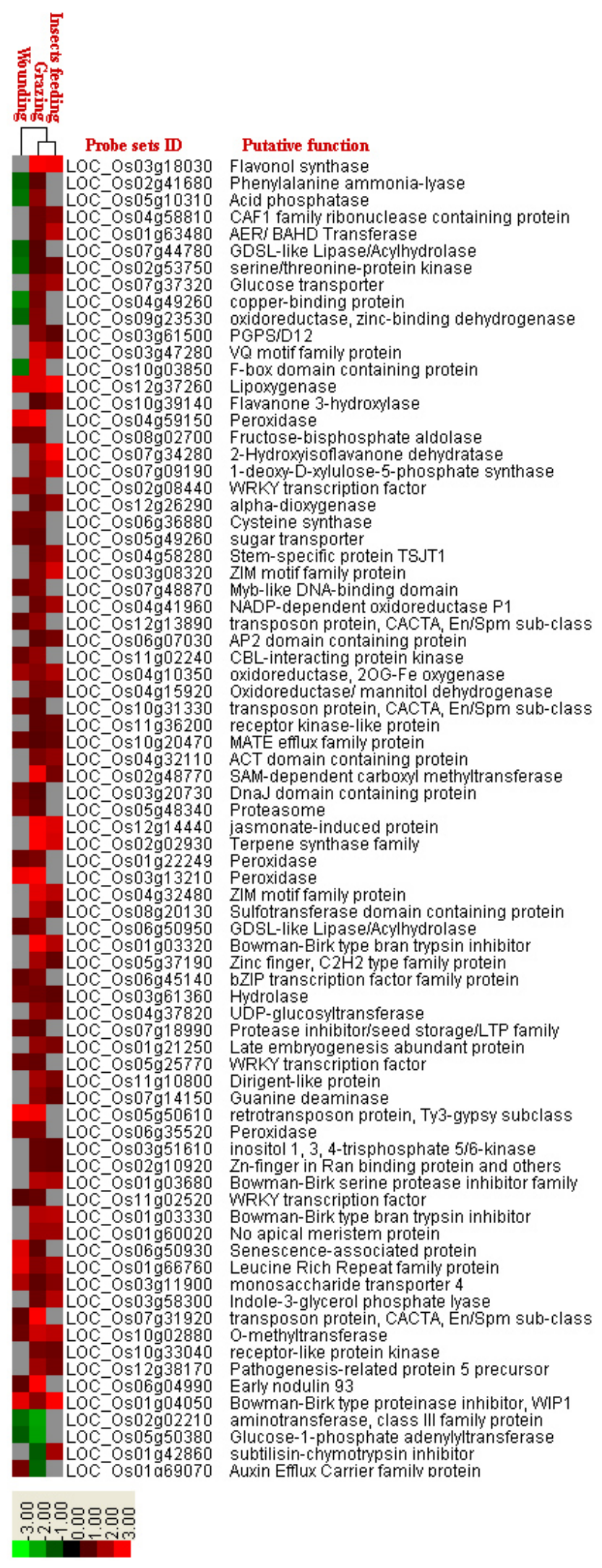

Figure 7. Comparative cluster analysis of the genes induced by biostresses. Data sources: grazing (this study), wounding (Lan et al., 2005), and insect feeding (Yuan et al., 2008). by insect feeding (Figure 7). Genes encoding subtilisinchymotrypsin inhibitor and metallothionein-like protein were found to exhibit rapidly and highly induced expression after insect feeding ( Yuan et al., 2005; Hua et al., 2007; Yuan et al., 2008), but down-regulated under grazing at $2 \mathrm{~h}$. Genes either shared by multiple stresses or specific to the grazing stress are subject to further investigation.

Plant cell walls play critical roles during the life cycle of a plant, including response to environmental stresses. In this study, a number of cell wall-related genes such as expansins and peroxidases were induced by grazing (Figure $3 \mathrm{C})$. These genes are involved in cell wall construction, modification, and maintenance of cell wall architecture. Expansins are involved in cell wall expansion (Cosgrove et al., 2002). Peroxidases play a wide range of functions in removal of peroxides, oxidation of toxic reductants, biosynthesis and degradation of lignin, suberization, and auxin catabolism. The peroxidase gene expression activity was up-regulated in response to environmental stresses such as wounding (Figure 7) and pathogen attacks (Tognolli et al., 2002).

Cytochrome P450s participate in many biochemical pathways, such as those for the synthesis/metabolism of phenylpropanoids, alkaloids, terpenoids, lipids and involve in the protection of plant against environmental stresses. P450 genes identified in this study can also activated by wounding (Yang et al., 2005) and insects feeding (Yang et al., 2006). In the current study, several secondary metabolic pathways, such as flavonoid and terpene pathways, were significantly induced after grazing. Flavonoids, synthesized via the phenylpropanoid pathway, are a diverse group of secondary metabolites with a wide array of biological functions, including the defense against various stresses, such as pathogens, wounding, and UV light damage (Winkel-Shirley, 2002). Terpene synthase gene, indole-3glycerol phosphate lyase gene, lipoxygenase gene, and phenylalanine ammonia-lyase gene induced by this study were involved in biosynthesis of plant volatiles of terpenoids, indole, fatty acid derivatives, and phenylpropanoids/ benzenoids. These genes were also responsive to insect feeding and/or wounding (Figure 7). Volatile terpenes are the most abundant armyworm induced volatile in rice (Yuan et al., 2008). Volatiles induced by cow saliva in plants need future investigation.

Signal transduction pathways target transcription factors and chromatin modifying factors leading to their phosphorylation by protein kinases or dephosphorylation in response to alterations in the environment. These modifications influence transcription factor activities and regulate gene expression networks, which results in appropriate changes in cell behavior (Hunter and Karin, 1992; Hill and Treisman, 1995). Grazing-induced activity changes of several signal transduction pathway genes such as protein kinases and many transcription factors such as AP2 and WRKY were observed in rice seedlings (Figure $3 A$ ). Most of these protein kinases and transcription factors were also reported to have response to insects feeding in rice (Figure 7). The $\mathrm{C}_{2} \mathrm{H}_{2}$-type zinc finger proteins represent a large and transcription factor family. These proteins play various roles in developmental processes. They also respond to multiple stresses including wounding, pathogen infection, and abiotic stresses and may be regulators in the ROS scavenging mechanism (Takatsuji, 1999; Davletova et 
Table 2. Known micro-RNA and its target genes responsive to grazing.

\begin{tabular}{lll}
\hline Probe sets ID & Gene product & Known micro-RNA accession \\
\hline Os.23468.1.A1_at & Avr9 elicitor response protein & MI0001050 \\
Os.50455.1.S1_at & Phospholipase D alpha 2 & MI0001439 \\
Os.36152.1.S1_at & Zinc finger, C2H2 type family protein & MI0001434 \\
Os.11608.1.S1_at & OsIAA6 - Auxin-responsive Aux/IAA gene family member & MI0001149 \\
Os.609.3.S1_a_at & Plastocyanin-like domain containing protein & MI0001149, MI0001434 \\
Os.6450.1.S1_at & 1-deoxy-D-xylulose-5-phosphate synthase & MI0001434 \\
Os.10091.1.S1_at & Long cell-linked locus protein & MI0001147 \\
Os.14921.1.S1_at & Protein app1 & MI0001704, MI0001705, MI0001706, MI0001718 \\
Os.10255.1.S1_s_at & F-box domain containing protein & MI0001049 \\
Os.24864.3.S1_at & Acid phosphatase & MI0001063 \\
OS.14830.1.S1_A_AT & Multicopper oxidase family protein & MI0001050 \\
OS.53763.1.A1_AT & Leucine Rich Repeat family protein & MI0001092, MI0001093
\end{tabular}

al., 2005; Vogel et al., 2005). AP2 gene families and WRKY proteins have been reported to be induced by wounding, SA, JA, ethylene, and pathogen attack (Rushton et al., 1996; Eulgem et al., 2000; Maleck et al., 2000; Schenk et al., 2000).

Among grazing induced genes, thirteen genes were found to be the targets of known miRNA (Table 2). These genes involved in signal transduction, transcription factor, and stress defense. It has been reported that miRNAs can be induced by environmental stress (Jones-Rhoades and Bartel, 2004; Sunkar and Zhu, 2004), suggesting that miRNAs may play an important role in regulating plant response to grazing.

\section{Carbohydrate metabolism}

The stimulation of genes involved in starch degradation detected in this study can be interpreted by the following hypothesis: the energy and sugar demand in sink tissues, such as the roots, took some time before it slowed down to a balanced level. The tissues met this demand by stimulating starch degradation within the first 2 hours after grazing. Twenty-four hours after grazing, the sucrose transporter was no longer upregulated, which may suggest that the sink demand slowed down and sucrose transporter proteins had reached their balanced requirement.

Starch degradation can be either through starch phosphorylases or amylases, depending on the plants and conditions. Starch phosphorylases are responsible for starch degradation in stored potato tubers (Sowokinos, 2001). However, enhanced alpha-amylase gene expression has been reported in rice cell culture (Ho et al., 2001), water-stressed barley leaves (Jacobsen et al., 1986), virus-infected tobacco leaves (Heitz et al., 1991), and wounded mung bean cotyledons (Koizuka et al., 1995). The microarray-based findings in the rice seedling in this study also indicated that starch degradation in the rice leaves after grazing was through the alpha amylase pathway, not the starch phosphorylase pathways (Figure 6).

The immediate reduction in expression of genes involved in the starch synthesis pathways as well as glucosephosphate transporters suggested that the supply of starch synthesis-required energy and substrates was suddenly reduced by grazing off the mature leaf blades (Figure 6). This result might suggest that the immature leaves at the
3- to 4-leaf stage in non-clipped rice seedlings not only use translocated sucrose for growth but also use some of the over-flowed sucrose from the top leaves to conduct starch synthesis. To the best of our knowledge, this finding has not been previously reported in plants.

Grazing suddenly reduce the stomata uptake of oxygen at the plant level because of the sudden removal of most leaf blades that were rich in stomata on the surfaces. This reduction of oxygen uptake might limit the power of mitochondria to emergently produce sufficient energy for the plants. Therefore, the up-regulation of the anaerobic respiration pathway observed in this study is likely an emergency approach for the seedlings to meet the demand for providing essential energy after the sudden loss of photosynthetic leaves. This result is intriguing and may suggest a positive role of anaerobic respiration for aboveground non-water-stressed leafy tissues.

The lactate dehydrogenase was up-regulated at $2 \mathrm{~h}$, then declined at $6 \mathrm{~h}$ and $24 \mathrm{~h}$, while the aldehyde dehydrogenase remained up-regulated during the 24-hour period (Table 1, Figure 4). These results suggest that the aldehyde dehydrogenase pathway is the main anaerobic respiration pathway in rice seedlings under grazing-induced stress, but the relatively more toxic aldehyde dehydrogenase pathway can be also needed when there is a sudden loss of energy supply.

This study focuses on identifying genes and pathways in early response to grazing. Further studies may be needed to investigate other factors such as grazing intervals, effects of different animals, saliva types, teeth sharpness, grazing severity, clipping methods, seedling stages, grass species, and grass growing conditions.

\section{Conclusions}

This study illustrated that grazing caused the rice seedlings to immediately adjust their gene expression profile within two hours. In the aboveground tissues, gene expression data suggest that starch synthesis in the seedlings slowed down, while starch degradation increased. Sucrose synthesis and transportation immediately enhanced likely due to the demand for sugars in the sink from the remained tissues, and then likely due to the re-growth of the shoot. Anaerobic respiration pathways were enhanced in leafy tissues in the air without obvious anoxic stress, likely in 
response to emergency demand for the supply of energy/ substrates. Gene expression data favors the possibility that there is starch synthesis in the immature leaves or the lower leaf portion in non-clipped rice seedlings using some of the over-flowed sucrose from the full functional leaf blades. The activity of signal transduction and hormone pathways suggest that both wound-related recovery and many abiotic stress pathway-shared genes are stimulated in grazed plants. Many genes induced by simulated grazing are shared by wound response and insect feeding response. This provides us a clue that animal feeding and insects feeding may have some common response mechanism in plants. The genes and pathways with significantly modified expression activities detected in this study but not shared with wounding or insect-feeding may represent grazingspecific genes and may provide insights into the plant specific response to grazing.

\section{Acknowledgements}

We are grateful to Man Su and Zhipeng Liu for their assistance in this research, Professor Shihua Shen for his useful discussion and Rebecca Griffiths for her critical reading of the manuscript. This research was supported by the National Basic Research Program of China (973, 2007CB108905), Natural Science Foundation of China (No. 30600370 and 30500106), and the Key Project of the Chinese Academy of Sciences (KSCX2-YW-N-50).

\section{References}

Agarwal, S., and Grover, A. (2006) Molecular biology, biotechnology and genomics of flooding-associated low $\mathrm{O} 2$ stress response in plants. Crit. Rev. Plant Sci. 25, 1-21.

Belesky, D. P., and Hill, N. S. (1997) Defoliation and leaf age influence on ergot alkaloids in tall fescue. Ann. Bot. 79, 259-264.

Brueland, B. A., Harmoney, K. R., Moore, K. J., George, J. R., and Brummer, E. C. (2003) Forage and grazing lands: Developmental morphology of smooth bromegrass growth following spring grazing. Crop Sci. 43, 1789-1796.

Cheong, Y. H., Chang, H.-S., Gupta, R., Wang, X., Zhu, T., and Luan, S. (2002) Transcriptional profiling reveals novel interactions between wounding, pathogen, abiotic stress, and hormonal responses in Arabidopsis. Plant Physiol. 129, 661-677.

Cosgrove, D. J., Li, L. C., Cho, H. T., Hoffmann-Benning, S., Moore, R. C., and Blecker, D. (2002) The growing world of expansins. Plant Cell Physiol. 43, 1436-1444.

Crawley, M. J. (1990) Rabbit grazing, plant competition and seedling recruitment in acid grassland. J. Appl. Ecol. 27, 803-820.

Davletova, S., Schlauch, K., Coutu, J., and Mittler, R. (2005) The zinc-finger protein Zat12 plays a central role in reactive oxygen and abiotic stress signaling in Arabidopsis. Plant Physiol. 139, 847-856.

De Vos, M., Van Oosten, V. R., Van Poecke, R. M. P., Van Pelt, J. A., Pozo, M. J., Mueller, M. J., Buchala, A. J., Métraux, J. P., Van Loon, L. C., Dicke, M., and Pieterse, C. M. J. (2005) Signal signature and transcriptome changes of Arabidopsis during pathogen and insect attack. Mol. Plant-Microbe Interact. 18, 923-937.

Del-Val, E., and Crawley, M. J. (2005) Are grazing increaser species better tolerators than decreasers? An experimental assessment of defoliation tolerance in eight British grassland species. J. Ecol. 93, 1005-1016.

Detling, J. K., Dyer, M. I., and Winn, D. T. (1979) Net photosynthesis, root respiration, and regrowth of Bouteloua gracilis following simulated grazing. Oecologia 41, 127-134.

Donaghy, D. J., and Fulkerson, W. J. (2002) The impact of defoliation frequency and nitrogen fertilizer application in spring on summer survival of perennial ryegrass under grazing in subtropical Australia. Grass Forage Sci. 57, 351-359.

Ecker, J. R. (1995) The ethylene signal transduction pathway in plants. Science 268, 667-675.

Eulgem, T., Rushton, P. J., Robatzek, S., and Somssich, I. E. (2000) The WRKY superfamily of plant transcription factors. Trends Plant Sci. 5, 199-206.

Felton, G. W., Bi, J. L., Mathews, M. C., Murphy, J. B., Korth, K., Wesley, S. V., Lamb, C., and Dixon, R. A. (1999) Crosstalk between the signal pathways for pathogen-induced systemic acquired resistance and grazing-induced insect resistance. In Novartis Foundation Symposium, Vol 223, pp 166-174.

Gibson, G. (2002) Microarrays in ecology and evolution: a preview. Mol. Ecol. 11, 17-24.

Givan, C. V. (1999) Evolving concepts in plant glycolysis: Two centuries of progress. Biol. Rev. Camb. Phil. Soc. 74, 277-309.

Goff, S. A., Ricke, D., Lan, T. H., Presting, G., Wang, R., Dunn, M., Glazebrook, J., Sessions, A., Oeller, P., Varma, H., Hadley, D., Hutchison. D., Martin. C., Katagiri. F., Lange, B.M., Moughamer, T., Xia, Y., Budworth, P., Zhong, J., Miguel, T., Paszkowski, U., Zhang, S., Colbert, M., Sun, W.L., Chen, L., Cooper, B., Park, S., Wood, T.C., Mao, L., Quail, P., Wing, R., Dean, R., Yu, Y., Zharkikh, A., Shen, R., Sahasrabudhe, S., Thomas, A., Cannings, R., Gutin, A., Pruss, D., Reid, J., Tavtigian, S., Mitchell, J., Eldredge, G., Scholl, T., Miller, R.M., Bhatnagar, S., Adey, N., Rubano, T., Tusneem, N., Robinson, R., Feldhaus, J., Macalma, T., Oliphant, A., and Briggs, S. (2002) A draft sequence of the rice genome (Oryza sativa L. ssp. japonica). Science 296, 92-100.

Goffard, N., and Weiller, G. (2007) GeneBins: A database for classifying gene expression data, with application to plant genome arrays. BMC Bioinfo. 8, 87.

Gold, W. G., and Caldwell, M. M. (1990) The effects of the spatial pattern of defoliation on regrowth of a tussock grass - III. Photosynthesis, canopy structure and light interception. Oecologia 82, 12-17.

Guan, Y., and Nothnagel, E. A. (2004) Binding of Arabinogalactan Proteins by Yariv Phenylglycoside Triggers Wound-Like Responses in Arabidopsis Cell Cultures. Plant Physiol. 135, 1346-1366.

Hagen, G., and Guilfoyle, T. (2002) Auxin-responsive gene expression: Genes, promoters and regulatory factors. Plant Mol. Biol. 49, 373-385.

Heitz, T., Geoffroy, P., Fritig, B., and Legrand, M. (1991) Two apoplastic $\alpha$-amylases are induced in tobacco by virus infection. Plant Physiol. 97, 651-656.

Hill, C. S., and Treisman, R. (1995) Transcriptional regulation by extracellular signals: Mechanisms and specificity. Cell 80, 199-211.

Ho, S. L., Chao, Y. C., Tong, W. F., and Yu, S. M. (2001) Sugar coordinately and differentially regulates growth- and stressrelated gene expression via a complex signal transduction 
network and multiple control mechanisms. Plant Physiol. $125,877-890$

Hua, H., Lu, Q., Cai, M., Xu, C., Zhou, D. X., Li, X., and Zhang, Q. (2007) Analysis of rice genes induced by striped stemborer (Chilo suppressalis) attack identified a promoter fragment highly specifically responsive to insect feeding. Plant Mol. Biol. 65, 519-530.

Hunter, T., and Karin, M. (1992) The regulation of transcription by phosphorylation. Cell 70, 375-387.

Jacobsen, J. V., Hanson, A. D., and Chandler, P. C. (1986) Water stress enhances expression of an a-amylase gene in barley leaves. Plant Physiol. 80, 350-359.

Jauregui, B. M., Celaya, R., Garcia, U., and Osoro, K. (2007) Vegetation dynamics in burnt heather-gorse shrublands under different grazing management with sheep and goats. Agroforest. Sys. 70, 103-111.

Jones-Rhoades, M. W., and Bartel, D. P. (2004) Computational identification of plant MicroRNAs and their targets, including a stress-induced miRNA. Mol. Cell 14, 787-799.

Kato-Noguchi, H., Ohashi, C., and Sasaki, R. (2003) Metabolic adaptation to flooding stress in upland and lowland rice seedlings. Acta Physiol. Plant. 25, 257-261.

Kim, K. M., Cho, S. K., Shin, S. H., Kim, G. T., Lee, J. H., Oh, B. J., Kang, K. H., Hong, J. C., Choi, J. Y., Shin, J. S., and Chung, Y. S. (2005) Analysis of differentially expressed transcripts of fungal elicitor- and wound-treated wild rice (Oryza grandiglumis). J. Plant Res. 118, 347-354.

Koizuka, N., Tanaka, Y., and Morohashi, Y. (1995) Expression of alpha-amylase in response to wounding in mung bean. Planta 195, 530-534.

Kursteiner, O., Dupuis, I., and Kuhlemeier, C. (2003) The Pyruvate decarboxylase 1 gene of Arabidopsis is required during anoxia but not other environmental stresses. Plant Physiol. 132, 968-978.

Lan, L., Li, M., Lai, Y., Xu, W., Kong, Z., Ying, K., Han, B., and Xue, Y. (2005) Microarray analysis reveals similarities and variations in genetic programs controlling pollination/ fertilization and stress responses in rice (Oryza sativa L.). Plant Mol. Biol. 59, 151-164.

Li, C., and Wong, W. H. (2001) Model-based analysis of oligonucleotide arrays: Expression index computation and outlier detection. Proc. Natl. Acad. Sci. U. S. A. 98, 31-36.

Li, C., and Wong, W. H. (2001) Model-based analysis of oligonucleotide arrays: model validation, design issues and standard error application. Genome biol. 2

Liscum, E., and Reed, J. W. (2002) Genetics of Aux/IAA and ARF action in plant growth and development. Plant Mol. Biol. 49, 387-400.

Maleck, K., Levine, A., Eulgem, T., Morgan, A., Schmid, J., Lawton, K. A., Dangl, J. L., and Dietrich, R. A. (2000) The transcriptome of Arabidopsis thaliana during systemic acquired resistance. Nat. Genet. 26, 403-410.

McConn, M., Creelman, R. A., Bell, E., Mullet, J. E., and Browse, J. (1997) Jasmonate is essential for insect defense in Arabidopsis. Proc. Natl. Acad. Sci. U. S. A. 94, 5473-5477.

McPherson, K., and Williams, K. (1998) The role of carbohydrate reserves in the growth, resilience, and persistence of cabbage palm seedlings (Sabal palmetto). Oecologia 117, 460-468.

Meers, T., and Adams, R. (2003) The impact of grazing by Eastern Grey Kangaroos (Macropus giganteus) on vegetation recovery after fire at Reef Hills Regional Park, Victoria. Ecol. Manage. Rest. 4, 126-132.

Moran, P. J., Cheng, Y., Cassell, J. L., and Thompson, G. A. (2002) Gene expression profiling of Arabidopsis thaliana in compatible plant-aphid interactions. Arch. Insect Biochem. Physiol. 51, 182-203.

Navarro, L., Dunoyer, P., Jay, F., Arnold, B., Dharmasiri, N., Estelle, M., Voinnet, O., and Jones, J. D. G. (2006) A plant miRNA contributes to antibacterial resistance by repressing auxin signaling. Science 312, 436-439.

Nowak, R. S., and Caldwell, M. M. (1984) A test of compensatory photosynthesis in the field: Implications for herbivory tolerance. Oecologia 61, 311-318.

Orodho, A. B., and Trlica, M. J. (1990) Clipping and long-term grazing effects on biomass and carbohydrate reserves of Indian ricegrass. J. Range Manage. 43, 52-57.

Pecetti, L., and Piano, E. (2002) Penetrance of creepingrootedness in clonal progenies of lucerne and observations on underground morphology of plants differing for this character. Euphytica 128, 35-45.

Reymond, P., Bodenhausen, N., Van Poecke, R. M. P., Krishnamurthy, V., Dicke, M., and Farmer, E. E. (2004) A conserved transcript pattern in response to a specialist and a generalist herbivore. Plant Cell 16, 3132-3147.

Reymond, P., Weber, H., Damond, M., and Farmer, E. E. (2000) Differential gene expression in response to mechanical wounding and insect feeding in Arabidopsis. Plant Cell 12, 707-719.

Rushton, P. J., Torres, J. T., Parniske, M., Wernert, P., Hahlbrock, K., and Somssich, I. E. (1996) Interaction of elicitor-induced DNA-binding proteins with elicitor response elements in the promoters of parsley PR1 genes. EMBO J. 15, 5690-5700.

Schenk, P. M., Kazan, K., Wilson, I., Anderson, J. P., Richmond, T., Somerville, S. C., and Manners, J. M. (2000) Coordinated plant defense responses in Arabidopsis revealed by microarray analysis. Proc. Natl. Acad. Sci. U. S. A. $97,11655-11660$.

Sowokinos, J. R. (2001) Biochemical and molecular control of cold-induced sweetening in potatoes. Am. J. Potato Res. 78, 221-236.

Stintzi, A., Weber, H., Reymond, P., Browse, J., and Farmer, E. E. (2001) Plant defense in the absence of jasmonic acid: The role of cyclopentenones. Proc. Natl. Acad. Sci. U. S. A. $98,12837-12842$.

Stotz, H. U., Koch, T., Biedermann, A., Weniger, K., Boland, W., and Mitchell-Olds, T. (2002) Evidence for regulation of resistance in Arabidopsis to Egyptian cotton worm by salicylic and jasmonic acid signaling pathways. Planta 214, 648-652.

Sunkar, R., and Zhu, J. K. (2004) Novel and stress regulated microRNAs and other small RNAs from Arabidopsis w inside box sign. Plant Cell 16, 2001-2019.

Takatsuji, H. (1999) Zinc-finger proteins: The classical zinc finger emerges in contemporary plant science. Plant Mol. Biol. 39, 1073-1078.

Thornburg, R. W., and Li, X. (1991) Wounding Nicotiana tabacum leaves causes a decline in endogenous indole3-acetic acid. Plant Physiol. 96, 802-805.

Tognolli, M., Penel, C., Greppin, H., and Simon, P. (2002) Analysis and expression of the class III peroxidase large gene family in Arabidopsis thaliana. Gene 288, 129-138.

Tusher, V. G., Tibshirani, R., and Chu, G. (2001) Significance 
analysis of microarrays applied to the ionizing radiation response. Proc. Natl. Acad. Sci. U. S. A. 98, 5116-5121.

Van Poecke, R. M. P., and Dicke, M. (2004) Indirect defence of plants against herbivores: Using Arabidopsis thaliana as a model plant. Plant Biol. 6, 387-401.

Voelckel, C., Weisser, W. W., and Baldwin, I. T. (2004) An analysis of plant-aphid interactions by different microarray hybridization strategies. Mol. Ecol. 13, 3187-3195.

Vogel, J. T., Zarka, D. G., Van Buskirk, H. A., Fowler, S. G., and Thomashow, M. F. (2005) Roles of the CBF2 and ZAT12 transcription factors in configuring the low temperature transcriptome of Arabidopsis. Plant J. 41, 195-211.

Vourch', G., Russell, J., and Martin, J. L. (2002) Linking deer browsing and terpene production among genetic identities in Chamaecyparis nootkatensis and Thuja plicata (Cupressaceae). Journal of Heredity 93(5), 370-376.

Winkel-Shirley, B. (2002) Biosynthesis of flavonoids and effects of stress. Curr. Opin. Plant Biol. 5, 218-223.

Yang, D. H., Chung, B. Y., Kim, J. S., Kim, J. H., Yun, P. Y., Lee, Y. K., Lim, Y. P., and Lee, M. C. (2005) cDNA cloning and sequence analysis of the rice cinnamate-4-hydroxylase gene, a cytochrome P450-dependent monooxygenase involved in the general phenylpropanoid pathway. J. Plant Biol. 48, 311-318.
Yang, Z., Zhang, F., Zhu, L., and He, G. (2006) Identification of differentially expressed genes in brown planthopper Nilaparvata lugens (Hemiptera: Delphacidae) responding to host plant resistance. Bull. Entomol. Res. 96, 53-59.

Yuan, H., Chen, X., Zhu, L., and He, G. (2005) Identification of genes responsive to brown planthopper Nilaparvata lugens Stål (Homoptera: Delphacidae) feeding in rice. Planta 221, 105-112.

Yuan, J. S., Köllner, T. G., Wiggins, G., Grant, J., Degenhardt, J., and Chen, F. (2008) Molecular and genomic basis of volatile-mediated indirect defense against insects in rice. Plant J. 55, 491-503.

Zhang, Y., Fan, W., Kinkema, M., Li, X., and Dong, X. (1999) Interaction of NPR1 with basic leucine zipper protein transcription factors that bind sequences required for salicylic acid induction of the PR-1 gene. Proc. Natl. Acad. Sci. U. S. A. 96, 6523-6528.

Zhou, J. M., Trifa, Y., Silva, H., Pontier, D., Lam, E., Shah, J., and Klessig, D. F. (2000) NPR1 differentially interacts with members of the TGA/OBF family of transcription factors that bind an element of the PR-1 gene required for induction by salicylic acid. Mol. Plant-Microbe Interact. 13, 191-202.

\section{Supplemental Material}

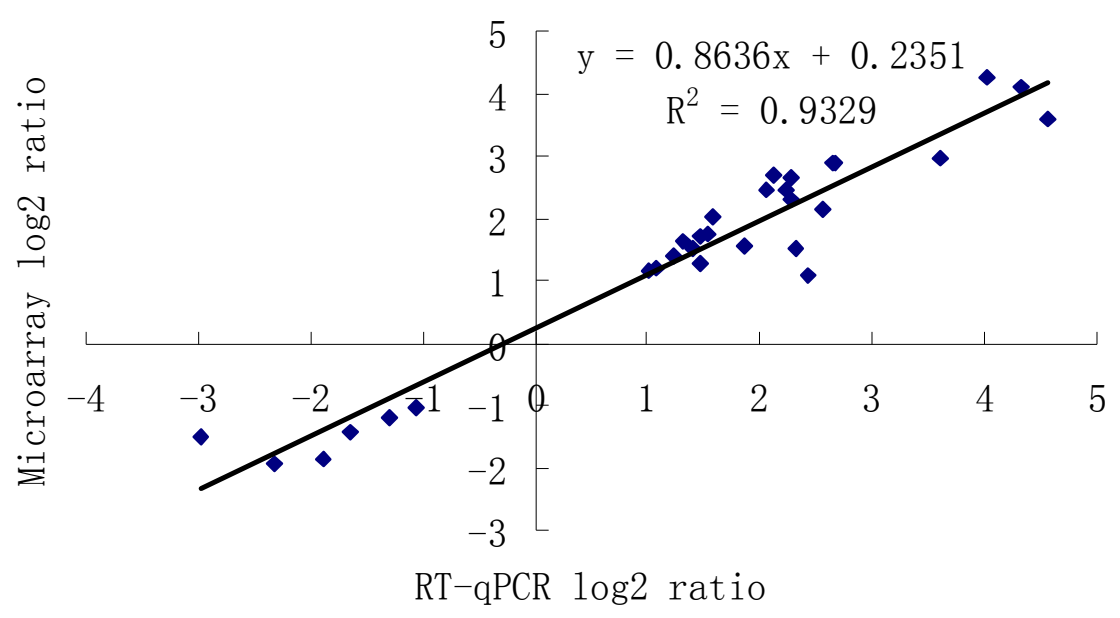

Figure S1. Correlation between microarray data and RT-qPCR data for the gene expression activities $2 \mathrm{~h}$ after grazing. Logtransformed fold change (base 2) for 30 differentially expressed genes. The gene IDs, products, and involved pathways and data regarding activity changes are presented in Table 1. 
Table S1. Genes and primers used in RT-qPCR analysis.

\begin{tabular}{|c|c|c|c|}
\hline Probe sets ID & Gene Product & Forward Primer (5'-3') & Reverse Primer (5'-3') \\
\hline Os.25606.1.S1 at & WRKY DNA binding domain protein & CAAGAAGAGGAGCAGGGAGA & CATTGGTACCCGTCCTTGAC \\
\hline Os.54934.1.S1_at & Myb-like DNA-binding domain protein & TGAGGAGGAGCACAGGCTAT & CAGTGATGTCGTGGATGCTC \\
\hline Os.27759.1.S1 at & Protein kinase domain containing protein & TGAGCTGTACGGTTCGACGGT & ACATCATCTGTCGGCGAGCC \\
\hline Os.15426.1.S1 at & Receptor-like protein kinase homolog RK20-1 & TCCTCAATGGCACGACGATG & TCTCTCGTCCCGGCGTGGCC \\
\hline Os.19617.1.S1 at & Leucine Rich Repeat family protein & CGCTCACTCGGTTTCGTTCAG & ACGGTGCACCACAAAGCCTT \\
\hline Os.19952.1.S1_at & $\begin{array}{l}\text { OsIAA2 - Auxin-responsive Aux/IAA gene } \\
\text { family member }\end{array}$ & GTCATCGTCGTCCAAGCAG & CGCCGTCCATGTTGATCT \\
\hline Os.11608.1.S1_at & $\begin{array}{l}\text { OsIAA6 - Auxin-responsive Aux/IAA gene } \\
\text { family member }\end{array}$ & GTGGAGGAAATGATAGCAGCCG & TCTTCCCTGGGTCTGTGGTCTG \\
\hline Os.17449.1.A1 at & Auxin efflux carrier component 6 & ATCTTCACGAGGGAGCAGTGCG & TTCACCTGGAACGGGTCGGT \\
\hline Os.31778.1.S1_x_at & Gibberellin 20 oxidase 2 & AGAGCCCGGCCAAATACTGG & ACAGCAACCTCATCAGCCGC \\
\hline Os.53660.1.S1 at & Ethylene responsive protein & AATCCGGCGTCGAGAGAGGT & ACCATGTCCTCGGCGTCGTT \\
\hline Os.405.1.S1 a at & Lipoxygenase 2.1 & CCACTGCAACTCTTGGATCG & TGCGCAGATTCTTCACGC \\
\hline Os.27112.1.S1_at & Regulatory protein NPR1 & CCCACAACTCGGAGAAGC & CGGAAACATAATCCTCGCC \\
\hline Os.54545.1.S1 at & Dihydroflavonol-4-reductase & CCCTCGCAGTACCAAGTACA & GATCGAGAGGAGTCCAGCAT \\
\hline Os.17112.1.S1 at & Flavonol synthase & CGCTGGCCTTCTTCTACAAC & GGTTGCCATCTGCTGAGACT \\
\hline Os. $10510.1 . \mathrm{S} 1$ at & Naringenin 3-dioxygenase & GTTCTCAAGGAAGGCAGGTG & CATCGGTGATGAGCTTCTGA \\
\hline Os.46511.1.S1_at & flavonol 3-O-methyltransferase & ATGATGCCAAGGATGGTAGC & GGGAGATCCAAAACCGTACA \\
\hline Os.18431.1.S1_at & chalcone synthase & TACTACCTCGTGCCGGTCTC & TCACCTTGGTCATGAACTCC \\
\hline Os.51127.1.S1 a at & flavonol 3-O-glucosyltransferase & GTGGCTTCCACATCACCTTC & AAGTGAGGGAGGCACGTCT \\
\hline Os.7314.1.S1 at & Glucan 1,3-beta-glucosidase & ACAAGTGGGCTAAACGCAGT & ACTGTGCTCAAACGGGTTCT \\
\hline Os.4179.1.S1_at & 1,4-alpha-glucan branching enzyme & GATGTTGTTCACAGCCATGC & TACTCCTCGAGCCACCATCT \\
\hline Os.47946.1.S1_s_at & Glucose-6-phosphate/phosphate translocator 2 & GCCAAGATCGGGGTCTACTT & GACAGGGCCTTCCAGAAATC \\
\hline Os.49091.1.S1 at & sucrose phosphate synthase & ATCGTTGTCGAGGAGACAGC & AATCCATAACTTCCCGAGCA \\
\hline Os.21893.3.A1 at & trehalose-6-phosphate synthase & CGAAACAACTGATGGGTCCT & CCTTGCTAACTCCCTGTGGT \\
\hline Os.9212.1.S1 at & Granule-bound starch synthase $1 \mathrm{~b}$ & GTCGAGTGCGAAACTGTTGA & CTTGAGCCATGCAGTTCTGA \\
\hline Os.4166.1.S1 at & Glucose-1-phosphate adenylyltransferase & TGGGAAGACATTGGAACGAT & CGATGGTACATTCACGCAAG \\
\hline Os.10908.1.S1_a_at & Alpha-amylase isozyme & GTGAAGATCGGGACGAGGTA & CCTTCTCCCAGACGCTGTAG \\
\hline Os.10215.1.S1_at & L-lactate dehydrogenase & GATGTCCAGGCGTACATGG & GAAGCTCTGGTGGCTCTCC \\
\hline Os.19003.1.S1 a at & Pyruvate dehydrogenase & CACACTCTCCACACCAGCAG & CCCCTTCCCTCCAGTAACTC \\
\hline Os.313.1.S1 a at & Aldehyde dehydrogenase & GGCGAGCAATTCAAGAAGAT & AGACGGTTGGCTGGATGTAG \\
\hline Os.21906.1.S1 at & Sugar transporter family protein & CGTGAACCAGTTGTCCGTTA & TCAAACCCCСТCAAAACTTG \\
\hline Os03g0718100- & actin (as RT-qPCR internal control) & ATGGAGACTGCCAAGACCAG & AAGGAAGGCTGGAAGAGGAC \\
\hline
\end{tabular}

Table S2. Differential expression genes in response to stimulated grazing at $2 \mathrm{~h}$ detected by microarray analysis (Attached MS Excel file). The list includes 376 up-regulated genes with log2 (ratio) $\geq 1$ (2-fold change) and 90 down-regulated genes with $\log 2$ (ratio) $\leq-1$ (0.5-fold change).

\begin{tabular}{|c|c|c|c|c|}
\hline Probe sets ID & $\begin{array}{l}\text { Fold } \\
\text { change }\end{array}$ & $\begin{array}{l}\log 2 \\
\text { ratio }\end{array}$ & Locus ID & Putative function \\
\hline OS.1478.1.S1_AT & 0.03 & -4.87 & LOC_Os03g19436.1 & Nicotianamine synthase 1 \\
\hline Os.51491.1.S1_at & 0.07 & -3.89 & LOC_Os11g21970.1 & transposon protein, putative, CACTA, En/Spm sub-class \\
\hline Os.409.1.S1_at & 0.10 & -3.35 & LOC_Os07g15460 & Metal transporter Nramp1 \\
\hline OS.9311.1.S1_AT & 0.11 & -3.14 & LOC_Os03g19420 & Nicotianamine synthase 2 \\
\hline Os.12498.1.S1_at & 0.12 & -3.10 & LOC_Os01g72370.2 & Helix-loop-helix DNA-binding domain containing protein \\
\hline Os.26063.1.S1_at & 0.12 & -3.01 & LOC_Os12g43060 & retrotransposon protein \\
\hline Os.49111.1.S1_at & 0.13 & -2.92 & LOC_Os04g48930 & ferric-chelate reductase \\
\hline Os.16044.1.S1_at & 0.14 & -2.82 & LOC_Os02g30910 & nodulin MtN3 family protein \\
\hline Os.14921.1.S1_at & 0.18 & -2.48 & LOC_Os03g56930 & expressed protein \\
\hline Os.12629.1.S2_at & 0.19 & -2.38 & LOC_Os03g64140.1 & expressed protein \\
\hline Os.54370.1.S1_at & 0.21 & -2.23 & LOC_Os03g48750 & Oxalate oxidase GF-2.8 precursor \\
\hline Os.4978.1.S2_at & 0.23 & -2.10 & LOC_Os03g54000 & oligopeptide transporter 3 \\
\hline Os.6330.1.s1_at & 0.23 & -2.10 & LOC_Os03g07180 & Late embryogenesis abundant protein \\
\hline OS.2821.1.A1_AT & 0.26 & -1.96 & LOC_Os02g02210 & aminotransferase, class III family protein \\
\hline Os.27759.1.S1__at & 0.26 & -1.94 & LOC_Os01g46720 & Protein kinase domain containing protein \\
\hline Os.9216.1.S1_at & 0.26 & -1.92 & LOC_Os03g26210 & Helix-loop-helix DNA-binding domain containing protein \\
\hline OS.51718.1.S̄_AT & 0.26 & -1.92 & LOC_Os11g26780 & Dehydrin RAB $16 B$ \\
\hline Os.11150.1.S1_at & 0.27 & -1.90 & LOC_Os03g32490 & expressed protein \\
\hline Os.4166.1.S1_at & 0.27 & -1.87 & LOC_Os05g50380 & Glucose-1-phosphate adenylyltransferase \\
\hline Os.54698.1.S1_at & 0.28 & -1.83 & LOC_Os03g51540 & Lariat debranching enzyme, C-terminal domain containing protein \\
\hline Os.32686.1.S1_at & 0.28 & -1.82 & LOC_Os01g65110 & POT family protein \\
\hline Os.52323.1.s1_at & 0.30 & -1.73 & LOC_Os12g15000 & retrotransposon protein, putative, Ty1-copia subclass \\
\hline Os.12551.1.S1_s_at & 0.30 & -1.72 & LOC_Os05g46480 & Late embryogenesis abundant protein \\
\hline OS.11491.1.S1_AT & 0.30 & -1.72 & LOC_Os03g19290 & $\begin{array}{l}\text { mitochondrial import inner membrane translocase subunit } \\
\text { Tim17/Tim22/Tim23 family protein }\end{array}$ \\
\hline Os.27943.1.S1_a_at & 0.31 & -1.71 & LOC_Os01g50030 & Phosphoethanolamine N-methyltransferase \\
\hline Os.55575.1.S1_at & 0.31 & -1.71 & LOC_Os05g02200 & $33 \mathrm{kDa}$ secretory protein \\
\hline Os.55491.1.s1_at & 0.31 & -1.70 & LOC_Os11g05550 & expressed protein \\
\hline
\end{tabular}




\begin{tabular}{|c|c|c|c|c|}
\hline Os.55380.1.S1_at & 0.31 & -1.68 & LOC_Os06g46740 & early nodulin 20 precursor \\
\hline Os.27606.1.S1_at & 0.32 & -1.66 & LOC_Os03g63330 & Aspartate kinase family protein \\
\hline Os.12633.1.s1_s_at & 0.32 & -1.65 & LOC_Os11g26790 & Dehydrin family protein \\
\hline Os.52298.1.S1_at & 0.32 & -1.64 & LOC_Os06g02960 & expressed protein \\
\hline Os.50950.1.S1_x_at & 0.33 & -1.61 & LOC_Os02g44730 & tetracycline transporter protein \\
\hline Os.28427.1.S2_a_at & 0.33 & -1.61 & LOC_Os05g12640 & polygalacturonase \\
\hline Os.55671.1.s1_at & 0.33 & -1.60 & LOC_Os06g44250 & Haemolysin-III related family protein \\
\hline Os.30528.1.S1_at & 0.33 & -1.59 & LOC_Os08g31860 & expressed protein \\
\hline OsAffx.10755.1.S1_at & 0.33 & -1.59 & LOC_Os03g46454.1 & zinc transporter 10 precursor \\
\hline Os.17916.1.S1_at ${ }^{-}$ & 0.33 & -1.58 & LOC_Os05g12630 & expressed protein \\
\hline OS.9417.1.S1_AT & 0.34 & -1.57 & LOC_Os09g36680 & Ribonuclease T2 family protein \\
\hline Os.9212.1.S1_at & 0.35 & -1.52 & LOC_Os07g22930 & granule-bound starch synthase $1 \mathrm{~b}$ \\
\hline OSAFFX.1873̄7.1.S1_AT & 0.35 & -1.50 & --- & -- \\
\hline OSAFFX.25073.1.S1_X_AT & 0.36 & -1.48 & LOC_Os03g12700 & expressed protein \\
\hline OS.34112.1.S1_AT -- & 0.36 & -1.47 & LOC_Os04g12700 & retrotransposon protein, putative, Ty1-copia subclass \\
\hline Os.49571.1.S1_at & 0.37 & -1.45 & LOC_Os03g15530 & expressed protein \\
\hline Os.21957.1.S1_at & 0.37 & -1.45 & LOC_Os10g02260 & POT family protein \\
\hline Os.10091.1.S1_at & 0.37 & -1.45 & LOC_Os03g19070 & long cell-linked locus protein \\
\hline Os.47946.1.S1_s_at & 0.37 & -1.44 & LOC_Os07g33910 & Glucose-6-phosphate/phosphate translocator 2 \\
\hline Os.12629.1.S1_at & 0.37 & -1.44 & LOC_Os02g49650.1 & expressed protein \\
\hline OS.12761.1.S1_AT & 0.38 & -1.41 & LOC_Os03g46060 & P21 protein \\
\hline Os.7767.1.S1_at & 0.38 & -1.41 & LOC_Os07g04930 & expressed protein \\
\hline Os.18485.1.s̄̄_s_at & 0.38 & -1.39 & LOC_Os03g52680 & expressed protein \\
\hline Os.34952.1.S1_at & 0.39 & -1.38 & LOC_Os01g55610 & POT family protein \\
\hline Os.51460.1.S1_at & 0.39 & -1.36 & LOC_Os02g33380 & pectinesterase inhibitor domain containing protein \\
\hline Os.27650.1.S1_at & 0.40 & -1.32 & LOC_Os07g43560 & Protein kinase domain containing protein \\
\hline OS.23151.1.S1_AT & 0.40 & -1.30 & LOC_Os01g13610 & NmrA-like family protein \\
\hline OS.23187.1.S1_AT & 0.41 & -1.29 & LOC_Os04g01470 & O-methyltransferase family protein \\
\hline OS.14951.1.S1_AT & 0.41 & -1.27 & LOC_Os08g39730 & Cytochrome P450 76C2 \\
\hline OS.10583.1.S1_S_AT & 0.42 & -1.25 & LOC_Os06g04920 & Zn-finger in Ran binding protein and others containing protein \\
\hline Os.11260.1.S1_at & 0.42 & -1.25 & LOC_Os05g31670 & plasma membrane associated protein \\
\hline Os.52036.1.S1_at & 0.43 & -1.23 & LOC_Os05g31020 & Eukaryotic peptide chain release factor subunit 1-2 \\
\hline OS.2423.1.S1_AT & 0.43 & -1.21 & LOC_Os12g36850 & Pathogenesis-related protein Bet v I family protein \\
\hline Os.6288.1.S1_at & 0.43 & -1.21 & LOC_Os08g31850 & expressed protein \\
\hline OS.50222.1.S1_AT & 0.44 & -1.20 & LOC_Os12g24800 & nine-cis-epoxycarotenoid dioxygenase 4 \\
\hline Os.17449.1.A1_at & 0.44 & -1.19 & LOC_Os01g69070 & Auxin Efflux Carrier family protein \\
\hline Os.55378.1.S1_at & 0.44 & -1.18 & LOC_Os09g34890 & expressed protein \\
\hline OS.9103.1.S1_ATT & 0.46 & -1.13 & LOC_Os09g09930 & heavy metal-associated domain containing protein \\
\hline OS.46450.1.S1̄_AT & 0.46 & -1.13 & LOC_Os10g34930 & secretory protein \\
\hline OS.11218.1.S1_AT & 0.46 & -1.12 & LOC_Os07g44440 & peroxiredoxin \\
\hline OS.1043.1.S1_ĀT & 0.46 & -1.11 & LOC_Os01g42860 & subtilisin-chymotrypsin inhibitor 2 \\
\hline Os.9321.1.S1_a_at & 0.47 & -1.10 & LOC_Os01g65100 & POT family protein \\
\hline Os.53579.1.s̄̄_èt & 0.47 & -1.09 & LOC_Os02g30240 & expressed protein \\
\hline OS.3415.1.S1_AT & 0.47 & -1.09 & LOC_Os10g39680 & Acidic $27 \mathrm{kDa}$ endochitinase precursor \\
\hline OS.34273.1.S̄̄_AT & 0.47 & -1.09 & LOC_Os01g71710 & Amino acid permease family protein \\
\hline Os.50292.1.S1_at & 0.47 & -1.08 & LOC_Os02g13560 & Sugar transporter type $2 a$ \\
\hline OS.56976.1.S1_AT & 0.47 & -1.08 & LOC_Os12g38320 & expressed protein \\
\hline Os.47446.1.S1_at & 0.47 & -1.08 & LOC_Os07g01090 & proline transporter \\
\hline Os.11354.1.S1_at & 0.47 & -1.08 & LOC_Os04g51460 & xyloglucan endotransglucosylase/hydrolase protein 15precursor \\
\hline Os.1479.1.S1_at & 0.48 & -1.07 & LOC_Os07g48980 & Nicotianamine synthase 3 \\
\hline Os.49648.1.s̄1_s_at & 0.48 & -1.06 & LOC_Os02g54140 & Hsp20/alpha crystallin family protein \\
\hline OS.52461.1.S1_AT & 0.48 & -1.06 & LOC_Os06g02850 & expressed protein \\
\hline OS.27688.1.A1_AT & 0.48 & -1.05 & LOC_Os04g21350 & flowering promoting factor-like 1 \\
\hline Os.29003.1.S1_at & 0.49 & -1.04 & LOC_Os01g49210 & expressed protein \\
\hline Os.20230.1.S1_at & 0.49 & -1.03 & LOC_Os11g37950 & Barwin, putative, expressed \\
\hline OS.21387.3.S1_AT & 0.49 & -1.03 & LOC_Os04g48670 & hypothetical protein \\
\hline OS.17496.1.S1_AT & 0.49 & -1.03 & LOC_Os12g16090 & expressed protein \\
\hline OS.9279.1.S1_ĀT & 0.49 & -1.02 & LOC_Os12g38270 & Metallothionein-like protein 1 \\
\hline OS.4179.1.S1_AT & 0.49 & -1.02 & LOC_Os02g32660.1 & 1,4-alpha-glucan branching enzyme \\
\hline OS.16532.1.S1̄_AT & 0.49 & -1.02 & LOC_Os03g52370 & expressed protein \\
\hline OS.23518.1.A1_AT & 0.49 & -1.02 & LOC_Os02g36190 & Cytochrome P450 71D7 \\
\hline OS.165.1.S1_AT̄ & 0.50 & -1.01 & LOC_Os12g36880 & Pathogenesis-related protein Bet v I family protein \\
\hline OS.55484.1.S1_AT & 0.50 & -1.00 & LOC_Os02g45620 & uncharacterized plant-specific domain TIGR01568 family protein \\
\hline OS.54229.1.S1_AT & 2.00 & 1.00 & LOC_Os04g36670 & expressed protein \\
\hline OS.28032.1.A1_AT & 2.00 & 1.00 & LOC_Os01g21070 & endoglucanase \\
\hline OS.21349.1.S1_AT & 2.01 & 1.00 & LOC_Os03g04020 & Rare lipoprotein A like double-psi beta-barrel containing protein \\
\hline OS.36283.1.S1_AT & 2.01 & 1.01 & LOC_Os12g25090 & expressed protein \\
\hline OS.55270.1.S1_S_AT & 2.01 & 1.01 & LOC_Os04g54210 & expressed protein \\
\hline OS.14830.1.S1_A_AT & 2.01 & 1.01 & LOC_Os01g63190 & Multicopper oxidase family protein \\
\hline OS.7631.1.S1_AT ${ }^{-}$ & 2.01 & 1.01 & --- & -- \\
\hline OS.26059.1.S1̄_X_AT & 2.02 & 1.01 & LOC_Os03g20730 & DnaJ domain containing protein \\
\hline OS.11711.1.S1_AT' & 2.02 & 1.01 & LOC_Os12g17880 & U-box domain containing protein \\
\hline OS.49529.1.S1_AT & 2.02 & 1.01 & LOC_Os06g43810 & expressed protein \\
\hline OS.31771.2.S1_X_AT & 2.02 & 1.02 & --- & -- \\
\hline OS.18145.1.S1_X_AT & 2.02 & 1.02 & LOC_Os03g57660 & glutamate carboxypeptidase II \\
\hline OS.27140.1.S1_A_AT & 2.03 & 1.02 & LOC_Os03g61910 & retrotransposon protein, putative, Ty1-copia subclass \\
\hline OS.20169.1.S1_AT & 2.03 & 1.02 & LOC_Os06g34730 & expressed protein \\
\hline OS.11271.1.S1_A_AT & 2.03 & 1.02 & LOC_Os11g14544.1 & expressed protein \\
\hline OS.13866.1.S1_AT & 2.03 & 1.02 & --- & --- \\
\hline OS.5434.1.S1_ĀT & 2.03 & 1.02 & LOC_Os08g26850 & expressed protein \\
\hline OS.37457.1.S1_AT & 2.03 & 1.02 & LOC_Os07g44780 & GDSL-like Lipase/Acylhydrolase family protein \\
\hline
\end{tabular}




\begin{tabular}{|c|c|c|c|c|}
\hline OS.46872.1.S1_AT & 2.04 & 1.03 & LOC_Os10g37160 & flavonoid 3-monooxygenase \\
\hline OS.49297.1.S1_AT & 2.04 & 1.03 & 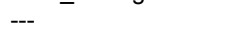 & --- \\
\hline OS.10884.1.S1_AT & 2.04 & 1.03 & LOC_Os03g58790 & ATPase 2 \\
\hline OS.22676.1.S1_AT & 2.04 & 1.03 & LOC_Os02g53750 & serine/threonine-protein kinase NAK \\
\hline OS.48287.1.S1_AT & 2.04 & 1.03 & LOC_Os04g38570 & multidrug resistance P-glycoprotein \\
\hline OS.5049.1.S1_AT & 2.04 & 1.03 & LOC_Os10g20470 & MATE efflux family protein \\
\hline OSAFFX.18575.1.S1_X_AT & 2.04 & 1.03 & LOC_Os10g39140 & naringenin 3-dioxygenase \\
\hline Os.18607.1.S1_at & 2.05 & 1.03 & LOC_Os09g29100 & Cyclin delta-2 \\
\hline OS.35020.1.S1_AT & 2.05 & 1.03 & LOC_Os11g03300 & NAC domain transcription factor \\
\hline OS.5354.1.S1_AT & 2.05 & 1.04 & LOC_Os12g06220 & Harpin-induced protein 1 containing protein \\
\hline OS.10880.1.S1_AT & 2.05 & 1.04 & LOC_Os04g45270 & Eukaryotic aspartyl protease family protein \\
\hline OS.50234.1.S1_AT & 2.06 & 1.04 & LOC_Os06g47930 & expressed protein \\
\hline OS.9086.1.S1_AT & 2.06 & 1.04 & LOC_Os03g10210 & Homeobox domain containing protein \\
\hline Os.19608.1.S1_at & 2.06 & 1.04 & LOC_Os09g38130 & Auxin Efflux Carrier family protein \\
\hline OS.1564.1.S1_AT & 2.06 & 1.05 & LOC_Os01g22380 & expressed protein \\
\hline OS.53828.1.S1_AT & 2.06 & 1.05 & LOC_Os08g41720 & Auxin Efflux Carrier family protein \\
\hline OS.53275.1.S1_AT & 2.07 & 1.05 & LOC_Os05g08420 & expressed protein \\
\hline OS.10162.1.S1_AT & 2.07 & 1.05 & LOC_Os01g28790 & expressed protein \\
\hline OS.26494.1.S1_AT & 2.07 & 1.05 & LOC_Os07g47400 & $\mathrm{C} 2$ domain containing protein \\
\hline Os.51231.1.S1_at & 2.08 & 1.05 & LOC_Os05g43910 & Cytochrome P450 71A1 \\
\hline OS.10619.1.S2_AT & 2.08 & 1.05 & LOC_Os05g44770 & Leucine Rich Repeat family protein \\
\hline OS.56891.1.S1_AT & 2.08 & 1.06 & LOC Os03g29930 & expressed protein \\
\hline OS.25243.1.A1_AT & 2.08 & 1.06 & LOC_Os03g20949.1 & Phospholipid-transporting ATPase 1 \\
\hline Os.47617.1.S1_at & 2.08 & 1.06 & LOC_Os04g52479.1 & expressed protein \\
\hline OS.11334.1.S1_X_AT & 2.09 & 1.06 & LOC_Os09g25090 & Protein kinase domain containing protein \\
\hline Os.10215.1.S1_at & 2.09 & 1.07 & LOC_Os02g01510 & L-lactate dehydrogenase A \\
\hline Os.8188.1.S1 s at & 2.09 & 1.07 & LOC_Os07g47620 & universal stress protein \\
\hline OS.55386.1.A1_AT & 2.10 & 1.07 & LOC_Os10g41330.1 & ethylene-responsive transcription factor 5 \\
\hline Os.46844.1.S1_at & 2.11 & 1.08 & LOC_Os10g25400 & Esterase precursor \\
\hline OS.27808.1.S1_S_AT & 2.11 & 1.08 & LOC_Os03g53200 & Calmodulin \\
\hline Os.54421.1.S1_at & 2.11 & 1.08 & LOC_Os05g37250 & Cytochrome P450 94A2 \\
\hline OS.7751.1.S1_AT & 2.11 & 1.08 & LOC_Os02g47660 & Helix-loop-helix DNA-binding domain containing protein \\
\hline OS.54549.1.S1_AT & 2.12 & 1.08 & LOC_Os08g36590 & expressed protein \\
\hline OS.12082.1.S1_AT & 2.12 & 1.08 & LOC Os04g39350 & heavy metal-associated domain containing protein \\
\hline Os.10754.1.S1_at & 2.12 & 1.09 & LOC_Os03g06520 & Sulfate transporter 3.1 \\
\hline OS.10860.2.S1_AT & 2.12 & 1.09 & LOC_Os04g44320 & transposon protein, putative \\
\hline OS.15815.2.S1 X AT & 2.12 & 1.09 & LOC Os08g01490 & flavonoid 3-monooxygenase \\
\hline OS.11802.1.S1_AT & 2.12 & 1.09 & LOC_Os03g51650 & expressed protein \\
\hline Os.609.3.S1_a at & 2.13 & 1.09 & LOC_Os08g37670 & Plastocyanin-like domain containing protein \\
\hline OS.6723.1.S1_AT & 2.13 & 1.09 & LOC_Os02g33330 & B-type cyclin, putative \\
\hline Os.50825.1.s1_s_at & 2.13 & 1.09 & LOC_Os05g39990 & Alpha-expansin 1 precursor \\
\hline OS.4458.1.S1_AT & 2.13 & 1.09 & LOC_Os06g03580 & Zinc finger, $\mathrm{C} 3 \mathrm{HC} 4$ type family protein \\
\hline OS.11575.3.S1_X_AT & 2.14 & 1.09 & LOC_Os08g40480 & transposon protein \\
\hline Os.8696.1.S1_x_at & 2.14 & 1.10 & LOC_Os08g02250 & IQ calmodulin-binding motif family protein \\
\hline OS.6207.1.S1_S_AT & 2.14 & 1.10 & LOC_Os06g41840 & NAD dependent epimerase/dehydratase family protein \\
\hline OS.11967.1.S1_AT & 2.15 & 1.10 & LOC_Os03g45170 & Amino acid permease family protein \\
\hline OS.46627.1.S1_AT & 2.15 & 1.10 & LOC Os $10 \mathrm{~g} 40640$ & Glycogenin \\
\hline Os.51929.1.S1_at & 2.15 & 1.10 & LOC_Os10g31330 & transposon protein, putative, CACTA, En/Spm sub-class \\
\hline OS.2321.1.S1_AT & 2.15 & 1.10 & LOC_Os08g09060 & Germin-like protein subfamily 1 member 11 precursor \\
\hline Os.57402.1.S1_at & 2.15 & 1.10 & LOC_Os05g33680.1 & hypothetical protein \\
\hline OS.25687.1.S1_AT & 2.15 & 1.10 & LOC_Os02g41680 & Phenylalanine ammonia-lyase \\
\hline Os.53795.1.S1_at & 2.15 & 1.11 & LOC_Os03g08840 & expressed protein \\
\hline OS.11287.1.S1_AT & 2.15 & 1.11 & LOC_Os06g50930 & Senescence-associated protein DIN1 \\
\hline Os.27232.1.S1_at & 2.15 & 1.11 & LOC_Os01g68650 & uncharacterized plant-specific domain TIGR01615 family protein \\
\hline OS.56144.1.S1_AT & 2.16 & 1.11 & LOC_Os03g64260 & AP2 domain containing protein \\
\hline Os.5594.1.S1_at & 2.16 & 1.11 & LOC_Os05g48340 & proteasome \\
\hline Os.11851.1.S1_at & 2.16 & 1.11 & LOC_Os03g11900 & Sugar carrier protein $\mathrm{C}$ \\
\hline OS.11565.1.S1_AT & 2.17 & 1.12 & LOC_Os10g02040 & Peroxidase 53 precursor \\
\hline OS.8682.1.S1_AT & 2.17 & 1.12 & LOC_Os $10 \mathrm{~g} 08780$ & expressed protein \\
\hline OS.27185.1.S1__X_AT & 2.17 & 1.12 & LOC_Os07g35750 & Protein kinase domain containing protein \\
\hline OS.55314.1.S1_AT & 2.18 & 1.12 & LOC_Os03g38350 & expressed protein \\
\hline OS.22312.3.A1_A AT & 2.18 & 1.13 & LOC_Os05g28740 & universal stress protein family protein \\
\hline OS.49819.1.S1_AT & 2.19 & 1.13 & LOC_Os11g36200 & receptor kinase \\
\hline OS.27807.1.S1_AT & 2.19 & 1.13 & LOC_Os03g01740 & expressed protein \\
\hline Os.4653.1.S1_at & 2.19 & 1.13 & LOC_Os01g03340 & Bowman-Birk type bran trypsin inhibitor \\
\hline Os.52355.1.S1_at & 2.19 & 1.13 & LOC_Os06g33880 & expressed protein \\
\hline Os.4804.1.S1_at & 2.19 & 1.13 & LOC_Os06g07030 & AP2 domain containing protein \\
\hline OS.51506.1.S1 AT & 2.20 & 1.13 & LOC Os03g20600 & expressed protein \\
\hline OS.27175.1.S1_A AT & 2.20 & 1.14 & LOC_Os07g12890 & ZIP zinc/iron transport family protein \\
\hline Os.24103.1.A1_at & 2.20 & 1.14 & LOC_Os07g18990 & Protease inhibitor/seed storage/LTP family protein \\
\hline OS.26858.1.S1_AT & 2.21 & 1.14 & LOC_Os02g38840 & Glucose-6-phosphate 1-dehydrogenase \\
\hline OS.52493.1.A1_AT & 2.22 & 1.15 & LOC Os03g22210 & expressed protein \\
\hline OS.51479.1.S1AT & 2.22 & 1.15 & LOC Os09g25720 & expressed protein \\
\hline OS.27755.1.S1_AT & 2.22 & 1.15 & LOC_Os07g35560 & glucan endo-1,3-beta-D-glucosidase \\
\hline OS.50366.1.S1_X_AT & 2.23 & 1.15 & LOC_Os10g26150 & expressed protein \\
\hline Os.12400.2.S1_x at & 2.23 & 1.15 & LOC_Os08g30020 & expressed protein \\
\hline OS.10505.1.S1 AT & 2.23 & 1.16 & LOC Os10g39010 & Protein kinase domain containing protein \\
\hline Os.11657.1.S1 at & 2.23 & 1.16 & LOC Os01g47760 & glutaredoxin family protein \\
\hline Os.14882.1.S1_at & 2.24 & 1.16 & LOC_Os11g02520 & WRKY DNA binding domain containing protein \\
\hline Os.21906.1.S1_at & 2.24 & 1.16 & LOC Os05g49260 & Sugar transporter family protein \\
\hline OS.55729.1.S1_AT & 2.24 & 1.16 & LOC_Os05g47950 & flavonol 3-O-glucosyltransferase \\
\hline
\end{tabular}




\begin{tabular}{|c|c|c|c|c|}
\hline Os.9554.1.S1_at & 2.24 & 1.16 & LOC_Os11g06770.1 & DNA binding protein \\
\hline Os.10635.1.s̄i_s_at & 2.24 & 1.17 & LOC_Os04g51880 & GHMP kinases putative ATP-binding protein \\
\hline OS.14125.1.S1_AT & 2.25 & 1.17 & LOC_Os09g35030 & DREB1A protein, putative \\
\hline OS.15197.1.S1_AT & 2.25 & 1.17 & LOC_Os03g51610 & inositol 1, 3, 4-trisphosphate 5/6-kinase \\
\hline Os.30670.1.S1_at & 2.25 & 1.17 & LOC_Os07g22730 & AP2 domain containing protein \\
\hline Os.51106.1.S1_at & 2.25 & 1.17 & LOC_Os06g14540 & Endoglucanase 1 \\
\hline Os.20700.1.S1_at & 2.25 & 1.17 & LOC_Os03g62760 & expressed protein \\
\hline OS.40018.1.S1_AT & 2.26 & 1.18 & LOC_Os05g45410 & HSF-type DNA-binding domain containing protein \\
\hline OS.11197.2.S1_X_AT & 2.27 & 1.18 & LOC_Os09g30414.1 & Eukaryotic aspartyl protease family protein \\
\hline Os.51323.1.S1_at & 2.27 & 1.18 & LOC_Os12g14540 & expressed protein \\
\hline Os.19003.1.S1_a_at & 2.27 & 1.19 & LOC_Os01g21160 & pyruvate dehydrogenase \\
\hline Os.37729.1.S1_s_at & 2.28 & 1.19 & LOC_Os01g03360 & Bowman-Birk type bran trypsin inhibitor \\
\hline Os.11277.1.S1_s_at & 2.28 & 1.19 & LOC_Os08g32970 & Annexin family protein \\
\hline Os.55682.1.S1_at & 2.28 & 1.19 & LOC_Os03g22820 & expressed protein \\
\hline Os.54829.1.S1_at & 2.29 & 1.20 & LOC_Os08g02220 & Endoglucanase 1 \\
\hline OS.37243.1.S1_AT & 2.29 & 1.20 & LOC_Os01g51670 & expressed protein \\
\hline OS.49818.1.S1_S_AT & 2.29 & 1.20 & LOC_Os07g35310 & Serine/threonine-protein kinase receptor precursor \\
\hline Os.37565.1.S1_x_at & 2.30 & 1.20 & LOC_Os05g25770 & WRKY DNA binding domain containing protein \\
\hline OS.9556.1.S1_ĀT & 2.30 & 1.20 & LOC_Os12g44060.1 & nodulin family protein \\
\hline Os.2371.1.S1_at & 2.30 & 1.20 & LOC_Os12g26290 & alpha-dioxygenase \\
\hline Os.55625.1.s̄̄_at & 2.30 & 1.20 & LOC_Os05g30454.1 & Thiamin pyrophosphokinase, catalytic domain containing protein \\
\hline OS.27243.1.S1_AT & 2.30 & 1.20 & LOC_Os11g38870 & Helix-loop-helix DNA-binding domain containing protein \\
\hline Os.7902.1.S1_at & 2.31 & 1.21 & LOC_Os06g46950 & EF-hand Ca2+-binding protein CCD1 \\
\hline Os.6009.3.S1_a_at & 2.31 & 1.21 & LOC_Os03g08470 & AP2 domain containing protein \\
\hline Os.33184.1.s1_êt & 2.32 & 1.21 & LOC_Os01g51870 & embryo-abundant protein \\
\hline Os.11125.1.S1_a_at & 2.32 & 1.21 & LOC_Os01g59690 & F-box family protein \\
\hline Os.10555.1.S1_a_at & 2.32 & 1.22 & LOC_Os02g10920 & Zinc finger, $\mathrm{C} 2 \mathrm{H} 2$ type family protein \\
\hline Os.36162.1.S1_s_at & 2.33 & 1.22 & LOC_Os01g61044.1 & Transmembrane amino acid transporter protein \\
\hline Os.26629.1.S1_a_at & 2.34 & 1.22 & LOC_Os11g14190 & expressed protein \\
\hline Os.9840.1.S1_at & 2.34 & 1.23 & LOC_Os10g17489.1 & flavonol 3-O-glucosyltransferase \\
\hline Os.7028.1.S1_at & 2.35 & 1.23 & LOC_Os02g45710 & Zinc finger, $\mathrm{C} 3 \mathrm{HC} 4$ type family protein \\
\hline Os.12391.1.s̄i_a_at & 2.35 & 1.23 & LOC_Os02g54820 & trehalose-6-phosphate synthase \\
\hline Os.7339.1.S1_at & 2.35 & 1.23 & LOC_Os10g31850 & $\mathrm{CHY}$ zinc finger family protein \\
\hline OS.12119.1.S1_AT & 2.35 & 1.23 & LOC_Os01g64470 & Harpin-induced protein 1 containing protein \\
\hline OS.48064.1.S1_AT & 2.35 & 1.23 & LOC_Os09g26780 & ZIM motif family protein \\
\hline OS.12018.1.S1_AT & 2.36 & 1.24 & LOC_Os07g42730 & EF hand family protein \\
\hline OS.46444.1.S1_AT & 2.36 & 1.24 & LOC_Os10g09200 & hypothetical protein \\
\hline Os.1993.1.S1_at & 2.36 & 1.24 & LOC_Os01g53730 & expressed protein \\
\hline OS.15917.1.S1_AT & 2.37 & 1.24 & LOC_Os04g41960 & NADP-dependent oxidoreductase P1 \\
\hline Os.52334.1.S1_at & 2.37 & 1.25 & LOC_Os07g10160.1 & flavonol 3-O-glucosyltransferase \\
\hline Os.52728.1.S1_at & 2.38 & 1.25 & LOC_Os04g37880 & expressed protein \\
\hline Os.34913.1.S1_at & 2.38 & 1.25 & LOC_Os01g55160 & expressed protein \\
\hline Os.36264.1.S1_at & 2.38 & 1.25 & LOC_Os01g53750 & glucan endo-1,3-beta-glucosidase 4 \\
\hline OS.52185.1.A1_AT & 2.39 & 1.26 & LOC_Os04g35790 & expressed protein \\
\hline Os.15138.1.S1_at & 2.40 & 1.26 & LOC_Os01g41900 & MCB2 protein \\
\hline OS.14079.1.S1_AT & 2.40 & 1.26 & LOC_Os03g58300 & Indole-3-glycerol phosphate lyase \\
\hline Os.37996.1.S1_at & 2.40 & 1.27 & LOC_Os07g43800 & EF hand family protein, expressed \\
\hline Os.27092.1.S1_at & 2.40 & 1.27 & LOC_Os07g08000 & Protein kinase domain containing protein \\
\hline OS.17356.1.A1_A_AT & 2.41 & 1.27 & LOC_Os11g15060 & SAM dependent carboxyl methyltransferase family protein \\
\hline Os.49091.1.S1_at & 2.41 & 1.27 & LOC_Os05g05270 & sucrose phosphate synthase \\
\hline OS.52648.1.S1_AT & 2.41 & 1.27 & LOC_Os04g35540 & Amino acid permease family protein \\
\hline Os.15633.1.S1_at & 2.41 & 1.27 & LOC_Os10g35460 & COBRA-like protein 2 precursor \\
\hline Os.8367.1.S1_ât & 2.42 & 1.27 & LOC_Os08g02490 & AT hook motif family protein \\
\hline Os.27156.1.s1__at & 2.42 & 1.28 & LOC_Os07g12800 & expressed protein \\
\hline Os.53423.1.S1_at & 2.43 & 1.28 & LOC_Os02g51640.1 & expressed protein \\
\hline Os.38346.1.S1_x_at & 2.43 & 1.28 & LOC_Os01g36350 & Cytochrome P450 71C4 \\
\hline Os.140.3.S1_x_at & 2.43 & 1.28 & LOC_Os04g58810 & CAF1 family ribonuclease containing protein \\
\hline Os.6423.1.s1_at & 2.44 & 1.29 & LOC_Os08g39330 & expressed protein \\
\hline Os.37834.1.S1̄_a_at & 2.44 & 1.29 & LOC_Os04g58280 & Stem-specific protein TSJT1 \\
\hline Os.10548.1.S1_s_at & 2.46 & 1.30 & LOC_Os02g55130 & Serine carboxypeptidase II precursor \\
\hline Os.5583.1.S1_at & 2.47 & 1.30 & LOC_Os11g40660 & universal stress protein family protein \\
\hline Os.23468.1.A $\overline{1} \_$at & 2.47 & 1.31 & LOC_Os09g27950 & Avr9 elicitor response protein \\
\hline Os.2237.1.S1_at & 2.47 & 1.31 & LOC_Os07g48010 & Peroxidase 2 \\
\hline OS.9574.1.S1_AT & 2.48 & 1.31 & LOC_Os02g26790.2 & expressed protein \\
\hline Os.18232.1.s̄̄_at & 2.48 & 1.31 & LOC_Os02g46850 & Oligopeptide transporter 4 \\
\hline Os.37603.1.S1_at & 2.50 & 1.32 & LOC_Os01g63480 & AER, putative, expressed \\
\hline Os.30886.1.S1_at & 2.50 & 1.32 & LOC_Os03g61360 & hydrolase, alpha/beta fold family protein \\
\hline OS.26922.1.S1_AT & 2.50 & 1.32 & LOC_Os02g04170 & L-aspartate oxidase family protein \\
\hline OS.6037.1.S1_ĀT & 2.50 & 1.32 & LOC_Os09g27580 & potassium uptake protein \\
\hline OS.49536.1.S1̄AT & 2.51 & 1.33 & LOC_Os08g09950 & Acyl-desaturase \\
\hline OS.51741.1.S1_AT & 2.51 & 1.33 & LOC_Os05g25920 & hypothetical protein \\
\hline Os.8266.1.A1_at & 2.53 & 1.34 & LOC_Os03g55800 & Cytochrome P450 74A1 \\
\hline Os.29933.1.s̄1_at & 2.54 & 1.34 & LOC_Os07g44910 & PrMC3, putative, expressed \\
\hline Os.9283.1.S1_at & 2.55 & 1.35 & LOC_Os02g33070 & hypothetical protein \\
\hline Os.53027.1.s1_at & 2.55 & 1.35 & LOC_Os06g36880 & Cysteine synthase \\
\hline Os.7674.1.S1_ât & 2.55 & 1.35 & LOC_Os06g19070 & Cytochrome P450 76C2 \\
\hline Os.53502.1.s̄i__at & 2.56 & 1.35 & LOC_Os04g44550 & coiled-coil-helix-coiled-coil-helix domain containing protein \\
\hline Os.22197.1.S1_at & 2.56 & 1.35 & LOC_Os06g20200 & serine hydrolase \\
\hline Os.4974.1.S1_at & 2.56 & 1.36 & LOC_Os11g31190 & nodulin MtN3 family protein \\
\hline Os.55407.1.s̄i_at & 2.56 & 1.36 & LOC_Os03g58500 & hypothetical protein \\
\hline Os.12342.1.S1_at & 2.57 & 1.36 & LOC_Os11g04420 & expressed protein \\
\hline
\end{tabular}




\begin{tabular}{|c|c|c|c|}
\hline Os.38278.1.S1_at & 2.57 & 1.36 & LOC_Os03g06850 \\
\hline Os.4999.1.S1_at & 2.57 & 1.36 & LOC_Os06g50230.1 \\
\hline Os.2867.1.A1_at & 2.58 & 1.37 & LOC_Os11g45740 \\
\hline Os.8778.1.S1_a_at & 2.58 & 1.37 & LOC_Os06g11280 \\
\hline Os.8276.1.S1_at & 2.58 & 1.37 & LOC_Os02g45160 \\
\hline OS.54878.1.S1_AT & 2.58 & 1.37 & LOC_Os04g14480.1 \\
\hline OS.55944.1.S1_AT & 2.60 & 1.38 & LOC_Os11g06150 \\
\hline Os.10197.1.S1_at & 2.60 & 1.38 & LOC_Os11g24060 \\
\hline OS.46444.1.S1_X_AT & 2.61 & 1.38 & LOC_Os04g02530.1 \\
\hline Os.50455.1.S1_at & 2.61 & 1.39 & LOC_Os06g40170 \\
\hline Os.52058.1.S1_at & 2.62 & 1.39 & LOC_Os04g46600 \\
\hline Os.487.1.S1_at & 2.62 & 1.39 & LOC_Os01g15340 \\
\hline Os.12096.4.S1_s_at & 2.63 & 1.39 & LOC_Os12g01530 \\
\hline Os.15688.1.S1_at & 2.63 & 1.39 & LOC_Os12g36110 \\
\hline OS.55293.1.S1_AT & 2.64 & 1.40 & LOC_Os06g05510 \\
\hline Os.6089.1.S1_at & 2.64 & 1.40 & LOC_Os04g15920 \\
\hline Os.49489.1.S1_at & 2.64 & 1.40 & LOC_Os11g07950 \\
\hline Os.46800.1.S2_a_at & 2.65 & 1.40 & LOC_Os10g33040 \\
\hline Os.11608.1.S1_at & 2.65 & 1.41 & LOC_Os01g53880 \\
\hline Os.7612.1.S1_at & 2.66 & 1.41 & LOC_Os03g60840 \\
\hline Os.16558.1.S1_at & 2.67 & 1.42 & LOC_Os01g73200 \\
\hline OS.51639.1.S1_X_AT & 2.68 & 1.42 & LOC_Os10g38040 \\
\hline Os.10579.1.S1_at & 2.68 & 1.42 & LOC_Os08g02700 \\
\hline OS.52147.1.S1_AT & 2.68 & 1.42 & LOC_Os11g30760 \\
\hline Os.55252.1.S1_at & 2.68 & 1.42 & LOC_Os04g49260 \\
\hline Os.18038.1.S1_at & 2.69 & 1.43 & LOC_Os03g06840.1 \\
\hline Os.34373.1.S1_at & 2.69 & 1.43 & LOC_Os12g12580 \\
\hline Os.4844.1.S1_at & 2.70 & 1.43 & LOC_Os05g06970 \\
\hline Os.26698.1.s1__a_at & 2.70 & 1.43 & LOC_Os01g19820 \\
\hline Os.1503.1.S1_at & 2.71 & 1.44 & LOC_Os06g45140 \\
\hline Os.48545.1.s1__at & 2.73 & 1.45 & LOC_Os04g53810 \\
\hline Os.8940.1.S1_at & 2.73 & 1.45 & LOC_Os06g12030 \\
\hline Os.53254.1.s̄̄_at & 2.74 & 1.45 & LOC_Os06g12210 \\
\hline Os.17722.1.S1_at & 2.77 & 1.47 & LOC_Os01g22249.1 \\
\hline Os.46081.1.S1_at & 2.77 & 1.47 & LOC_Os10g13850 \\
\hline Os.11546.1.S1_at & 2.78 & 1.48 & LOC_Os06g35520 \\
\hline Os.11335.3.S1_x_at & 2.78 & 1.48 & LOC_Os01g52260 \\
\hline Os.20333.1.S1_at & 2.79 & 1.48 & LOC_Os02g55600 \\
\hline Os.19553.1.S1_at & 2.79 & 1.48 & LOC_Os05g49420 \\
\hline Os.11952.1.S1_at & 2.81 & 1.49 & LOC_Os02g11070 \\
\hline OS.4416.1.S1_AT & 2.81 & 1.49 & LOC_Os08g39840 \\
\hline Os.17761.2.S1_x_at & 2.81 & 1.49 & LOC_Os04g32110 \\
\hline Os.27112.1.S1_at & 2.82 & 1.50 & LOC_Os01g09800 \\
\hline Os.11296.1.s1_a_at & 2.82 & 1.50 & LOC_Os09g11460 \\
\hline Os.32357.1.S1_at & 2.82 & 1.50 & LOC_Os07g46950 \\
\hline Os.51959.1.s2_at & 2.84 & 1.51 & LOC_Os04g34420 \\
\hline Os.9900.1.S1_āt & 2.84 & 1.51 & LOC_Os04g22380 \\
\hline Os.52616.1.A $\overline{1} \_$at & 2.85 & 1.51 & LOC_Os03g29410 \\
\hline Os.27292.1.A1_at & 2.87 & 1.52 & LOC_Os04g44354.2 \\
\hline Os.51134.1.S1_at & 2.87 & 1.52 & LOC_Os07g10190 \\
\hline Os.54412.1.S1_at & 2.87 & 1.52 & LOC_Os04g35140 \\
\hline Os.7314.1.S1_at & 2.88 & 1.52 & LOC_Os10g22520 \\
\hline Os.49582.1.s1̄_at & 2.88 & 1.52 & LOC_Os02g45930 \\
\hline Os.5983.1.S1_at & 2.88 & 1.53 & LOC_Os09g23530 \\
\hline OS.46849.1.S1_AT & 2.89 & 1.53 & LOC_Os10g25230 \\
\hline Os.34496.1.S1_at & 2.89 & 1.53 & LOC_Os03g61500 \\
\hline Os.36152.1.S1_at & 2.92 & 1.54 & LOC_Os01g63980 \\
\hline Os.54932.1.s1_at & 2.92 & 1.54 & LOC_Os12g07030 \\
\hline Os.10356.1.S1_at & 2.92 & 1.55 & LOC_Os03g08320 \\
\hline Os.5066.1.S1_at & 2.92 & 1.55 & LOC_Os01g12000 \\
\hline Os.32634.1.s 1 _at & 2.92 & 1.55 & LOC_Os07g48870 \\
\hline Os.53237.1.S1_at & 2.93 & 1.55 & LOC_Os07g34070 \\
\hline Os.19617.1.S1_at & 2.93 & 1.55 & LOC_Os01g66760 \\
\hline Os.26932.1.S1_at & 2.94 & 1.56 & LOC_Os07g37320 \\
\hline Os.27251.1.S1_at & 2.95 & 1.56 & LOC_Os04g10350 \\
\hline Os.7095.1.S1_ât & 2.96 & 1.57 & LOC_Os04g49210 \\
\hline Os.6321.1.S1_at & 2.96 & 1.57 & LOC_Os01g63690 \\
\hline Os.27247.1.s1̄_at & 2.99 & 1.58 & LOC_Os06g50950 \\
\hline Os.26626.1.S1_at & 2.99 & 1.58 & LOC_Os05g33400 \\
\hline Os.12032.1.S1_at & 3.02 & 1.60 & LOC_Os02g08440 \\
\hline OS.53763.1.A1_AT & 3.03 & 1.60 & LOC_Os06g38340 \\
\hline OS.55595.1.S1_AT & 3.04 & 1.60 & LOC_Os12g02060 \\
\hline Os.5698.1.S1_s_at & 3.06 & 1.61 & LOC_Os05g33700 \\
\hline OS.55343.1.S1_AT & 3.07 & 1.62 & LOC_Os04g56030 \\
\hline Os.53660.1.S1_at & 3.08 & 1.62 & LOC_Os02g43790 \\
\hline Os.15191.1.s1_s_at & 3.10 & 1.63 & LOC_Os05g41220 \\
\hline Os.23405.1.s1'at & 3.11 & 1.64 & LOC_Os07g14150 \\
\hline Os.51029.1.S1_at & 3.12 & 1.64 & LOC_Os09g02770 \\
\hline Os.9739.1.S1_at & 3.13 & 1.64 & LOC_Os04g05650 \\
\hline Os.3729.1.S1_at & 3.13 & 1.65 & LOC_Os01g04050 \\
\hline Os.46941.1.s $1 \mathrm{~s}$ at & 3.13 & 1.65 & LOC_Os06g48500 \\
\hline
\end{tabular}

B3 DNA binding domain containing protein expressed protein expressed protein

12-oxophytodienoate reductase 2

Uncharacterized protein family UPF0005 containing protein hypothetical protein

expressed protein

xanthine/uracil/vitamin $\mathrm{C}$ permease family protein

expressed protein

Phospholipase D alpha 2

expressed protein

FPF1 protein

Ferritin 1, chloroplast precursor

calmodulin-binding protein

expressed protein

mannitol dehydrogenase

expressed protein

Leucine Rich Repeat family protein

AUX/IAA family protein

Bowman-Birk serine protease inhibitor family protein

Peroxidase 12

LysM domain containing protein

Fructose-bisphosphate aldolase

expressed protein

copper-binding protein

GRF zinc finger family protein

NADP-dependent oxidoreductase P2

Peroxidase 3 precursor

universal stress protein family protein

bZIP transcription factor family protein

Dihydroflavonol-4-reductase

Domain found in Dishevelled, Egl-10, and Pleckstrin family protein

bHLH family protein

Peroxidase family protein

expressed protein

Peroxidase 52

serine $\mathrm{O}$-acetyltransferase family protein

expressed protein

G-box binding factor 1

chalcone synthase

Lipoxygenase 7

ACT domain containing protein

Regulatory protein NPR1

AP2 domain containing protein

transposon protein, putative

Serine/threonine-protein kinase receptor precursor

retinol dehydrogenase 14

Serine/threonine-protein kinase NAK

flavonol 3-O-glucosyltransferase

flavonol 3-O-glucosyltransferase

Subtilisin N-terminal Region family protein

Glucan 1,3-beta-glucosidase

expressed protein

oxidoreductase, zinc-binding dehydrogenase family protein

ZIM motif family protein

PGP224, putative, expressed

Zinc finger, $\mathrm{C} 2 \mathrm{H} 2$ type family protein

expressed protein

ZIM motif family protein

expressed protein

Myb-like DNA-binding domain containing protein

Integral membrane protein DUF6 containing protein

Leucine Rich Repeat family protein

Glucose transporter

oxidoreductase, 2OG-Fe oxygenase family protein

Naringenin 3-dioxygenase

Nematode-resistance protein

GDSL-like Lipase/Acylhydrolase family protein

Basic 7S globulin precursor

WRKY transcription factor

Leucine Rich Repeat family protein

Cationic peroxidase 1 precursor, putative

$4 \mathrm{~F} 5$ protein family protein

expressed protein

Ethylene responsive protein

SNF1-related protein kinase regulatory beta subunit 1

Guanine deaminase

expressed protein

expressed protein

Bowman-Birk type wound-induced proteinase inhibitor WIP1 precursor

expressed protein 


\begin{tabular}{|c|c|c|c|}
\hline Os.55494.1.S1_at & 3.14 & 1.65 & LOC_Os12g05690 \\
\hline Os.31185.1.S1_at & 3.15 & 1.65 & LOC_Os01g13950 \\
\hline Os.49352.1.S1_at & 3.15 & 1.66 & LOC_Os11g15570 \\
\hline Os.32455.1.S1_at & 3.17 & 1.66 & LOC_Os01g54670 \\
\hline Os.11778.1.S1_a_at & 3.17 & 1.66 & LOC_Os03g58580 \\
\hline Os.54944.1.S1_at & 3.18 & 1.67 & LOC_Os02g52670 \\
\hline Os.12063.1.S1_at & 3.19 & 1.67 & LOC_Os05g08830.1 \\
\hline Os.24864.3.S1_at & 3.20 & 1.68 & LOC_Os05g10310.2 \\
\hline OS.28514.1.S1_AT & 3.20 & 1.68 & LOC_Os01g04280 \\
\hline Os.6450.1.S1_at & 3.21 & 1.68 & LOC_Os07g09190 \\
\hline Os.51606.1.s1__at & 3.21 & 1.68 & LOC_Os12g43870. \\
\hline Os.31858.1.S1_at & 3.22 & 1.69 & LOC_Os07g35340 \\
\hline Os.53217.1.S1_x_at & 3.23 & 1.69 & LOC_Os02g45910 \\
\hline Os.9836.1.S1_at & 3.26 & 1.71 & LOC_Os11g10590 \\
\hline Os.51063.1.S1_at & 3.27 & 1.71 & LOC_Os09g28210 \\
\hline Os.51127.1.S1_a_at & 3.29 & 1.72 & LOC_Os04g37820 \\
\hline Os.26695.1.s1_at & 3.31 & 1.73 & LOC_Os03g60080 \\
\hline Os.25589.1.S1_at & 3.31 & 1.73 & LOC_Os11g25780 \\
\hline Os.313.1.S1_a_at & 3.31 & 1.73 & LOC_Os02g49720 \\
\hline Os.2915.1.S1̄_at & 3.33 & 1.74 & LOC_Os01g21250 \\
\hline Os.26802.1.S1_at & 3.34 & 1.74 & LOC_Os12g08130 \\
\hline Os.54936.1.S1_at & 3.36 & 1.75 & LOC_Os03g57640 \\
\hline Os.6662.1.S1_at & 3.36 & 1.75 & LOC_Os05g44900 \\
\hline Os.56145.1.S1_at & 3.38 & 1.76 & LOC_Os12g13890 \\
\hline Os.8118.1.S1_at & 3.38 & 1.76 & LOC_Os07g26630 \\
\hline Os.11923.1.S1_x_at & 3.39 & 1.76 & LOC_Os05g33630 \\
\hline Os.51546.1.S1_at & 3.44 & 1.78 & LOC_Os03g08520 \\
\hline Os.12012.1.S1_at & 3.44 & 1.78 & LOC_Os10g25290 \\
\hline Os.51753.1.A1_at & 3.46 & 1.79 & LOC_Os12g35340 \\
\hline Os.17677.1.S1_at & 3.46 & 1.79 & LOC_Os06g03810 \\
\hline OS.57361.1.S1_AT & 3.47 & 1.80 & LOC_Os08g13400 \\
\hline Os.49077.1.A1_at & 3.47 & 1.80 & LOC_Os03g45390 \\
\hline Os.15298.1.S1_at & 3.50 & 1.81 & LOC_Os03g19990 \\
\hline Os.50383.1.S1_at & 3.53 & 1.82 & LOC_Os06g05430 \\
\hline Os.4159.1.S1_at & 3.60 & 1.85 & LOC_Os01g51570 \\
\hline Os.50790.1.S1__at & 3.60 & 1.85 & LOC_Os03g52320 \\
\hline Os.12257.1.S1_at & 3.64 & 1.87 & LOC_Os02g52150 \\
\hline Os.4384.1.S1_at & 3.65 & 1.87 & LOC_Os01g60020 \\
\hline Os.6776.1.S1_at & 3.68 & 1.88 & LOC_Os07g35480 \\
\hline Os.50903.1.S1_at & 3.70 & 1.89 & LOC_Os09g08130 \\
\hline OS.17108.1.S1_AT & 3.71 & 1.89 & LOC_Os12g38170 \\
\hline Os.51147.1.S1_at & 3.71 & 1.89 & LOC_Os03g16960 \\
\hline Os.50399.1.S1_at & 3.74 & 1.90 & LOC_Os06g05420 \\
\hline Os.54232.1.S1_at & 3.75 & 1.91 & LOC_Os05g37190 \\
\hline Os.4453.1.S1_at & 3.79 & 1.92 & LOC_Os06g03520 \\
\hline Os.38309.1.s1̄_at & 3.81 & 1.93 & LOC_Os06g36390 \\
\hline Os.1310.1.S1_a_at & 3.83 & 1.94 & LOC_Os11g02240 \\
\hline OS.55961.1.S1_AT & 3.84 & 1.94 & LOC_Os07g34280 \\
\hline Os.57156.1.S1_at & 3.86 & 1.95 & LOC_Os11g10800 \\
\hline Os.48082.1.S1_at & 3.89 & 1.96 & LOC_Os09g25070 \\
\hline Os.11330.1.S2_at & 3.92 & 1.97 & LOC_Os03g28260 \\
\hline Os.53105.1.S1_at & 3.94 & 1.98 & LOC_Os11g04140 \\
\hline Os.27490.1.A1_at & 3.95 & 1.98 & $----(-\infty)$ \\
\hline Os.27675.1.S1_at & 4.03 & 2.01 & LOC_Os11g28940 \\
\hline Os.11961.1.S1_at & 4.05 & 2.02 & LOC_Os05g38040 \\
\hline Os.15426.1.S1_at & 4.09 & 2.03 & LOC_Os07g35370 \\
\hline Os.18406.1.S1_at & 4.17 & 2.06 & LOC_Os07g40220 \\
\hline Os.19472.1.S1_at & 4.27 & 2.10 & LOC_Os01g03330 \\
\hline Os.32459.1.S1_at & 4.38 & 2.13 & LOC_Os01g03310 \\
\hline Os.46511.1.S1_at & 4.42 & 2.14 & LOC_Os10g02840 \\
\hline Os.5045.1.S1_at & 4.44 & 2.15 & LOC_Os01g03680 \\
\hline Os.18404.1.S1_at & 4.46 & 2.16 & LOC_Os02g30780. \\
\hline Os.29923.1.S1_at & 4.47 & 2.16 & LOC_Os06g36380 \\
\hline Os.53936.1.S1_at & 4.47 & 2.16 & LOC_Os03g15270 \\
\hline Os.51923.1.S1_at & 4.53 & 2.18 & LOC_Os12g05440 \\
\hline Os.50805.1.S1_at & 4.56 & 2.19 & LOC_Os08g20130 \\
\hline Os.55511.1.S1_at & 4.58 & 2.20 & LOC_Os04g45520 \\
\hline Os.50057.1.S1_at & 4.64 & 2.21 & LOC_Os03g42200 \\
\hline Os.27271.1.S1_a_at & 4.65 & 2.22 & LOC_Os04g25060 \\
\hline Os.20206.1.S1_at & 4.68 & 2.23 & LOC_Os02g41840 \\
\hline Os.50180.1.S1_at & 4.88 & 2.29 & LOC_Os05g05100 \\
\hline Os.264.1.S1_at & 4.88 & 2.29 & LOC_Os04g59260 \\
\hline OS.55984.1.S1_AT & 4.89 & 2.29 & LOC_Os05g46840 \\
\hline Os.31088.1.S1_at & 4.91 & 2.30 & LOC_Os01g27140 \\
\hline OS.18431.1.S1_AT & 4.96 & 2.31 & LOC_Os09g34930 \\
\hline Os.37876.1.S1_at & 5.01 & 2.33 & LOC_Os10g39920 \\
\hline Os.158.1.S1_at & 5.16 & 2.37 & LOC_Os10g02070 \\
\hline Os.170.1.S1_at & 5.29 & 2.40 & LOC_Os01g64110 \\
\hline Os.7507.1.S1__at & 5.29 & 2.40 & LOC_Os10g02880 \\
\hline Os.20863.1.S1__at & 5.33 & 2.41 & LOC_Os01g62970 \\
\hline Os.52678.1.S1_at & 5.39 & 2.43 & LOC_Os04g41130 \\
\hline
\end{tabular}

expressed protein

Glutaredoxin-like family protein

expressed protein

expressed protein

nodulin family protein

AP2 domain containing protein

hypothetical protein

Acid phosphatase

calmodulin-binding protein

1-deoxy-D-xylulose-5-phosphate synthase

hypothetical protein

Receptor-like serine-threonine protein kinase

Naringenin 3-dioxygenase

hypothetical protein

Helix-loop-helix DNA-binding domain containing protein

flavonol 3-O-glucosyltransferase

NAC-domain containing protein 19

PB1 domain containing protein

Aldehyde dehydrogenase

Late embryogenesis abundant protein

amino acid transporter

PrMC3, putative, expressed

expressed protein

transposon protein, putative, CACTA, En/Spm sub-class

Aquaporin PIP2.2

inosine-uridine preferring nucleoside hydrolase family protein

expressed protein

ZIM motif family protein

Glutaredoxin-like family protein

expressed protein

hypothetical protein

Glucan endo-1,3-beta-glucosidase 5

WD40-like Beta Propeller Repeat family protein

expressed protein

Glucan endo-1,3-beta-glucosidase GII

SSXT protein containing protein

Hsp20/alpha crystallin family protein

No apical meristem protein

glucan endo-1,3-beta-glucosidase 3

Indole-3-glycerol phosphate synthase

Pathogenesis-related protein 5 precursor

$33 \mathrm{kDa}$ secretory protein

expressed protein

Zinc finger, $\mathrm{C} 2 \mathrm{H} 2$ type family protein

expressed protein

expressed protein

CBL-interacting serine/threonine-protein kinase 15

2-Hydroxyisoflavanone dehydratase

Dirigent-like protein

WRKY DNA binding domain containing protein

Late embryogenesis abundant protein

Integral membrane protein DUF6 containing protein

expressed protein

expressed protein

Receptor-like protein kinase homolog RK20-1

expressed protein

Bowman-Birk type bran trypsin inhibitor

Bowman-Birk type bran trypsin inhibitor

flavonol 3-O-methyltransferase

Bowman-Birk serine protease inhibitor family protein

LGC1, putative, expressed

expressed protein

expressed protein

Cytochrome P450 94A2

Sulfotransferase domain containing protein

Integral membrane protein

Dof domain, zinc finger family protein

expressed protein

expressed protein

expressed protein

Peroxidase 12 precursor

expressed protein

Glutaredoxin-like family protein

chalcone synthase

expressed protein

Peroxidase N precursor

Hevamine A precursor

flavonol 3-O-methyltransferase

expressed protein

FLOWERING LOCUS T protein 
100 Chen et al.

\begin{tabular}{|c|c|c|c|c|}
\hline Os.19952.1.S1_at & 5.41 & 2.44 & LOC_Os01g09450 & AUX/IAA family protein \\
\hline Os.21893.3.A1_at & 5.45 & 2.45 & LOC_Os12g32130 & trehalose-6-phosphate synthase \\
\hline Os.56080.1.S1_at & 5.56 & 2.47 & LOC_Os03g47280 & VQ motif family protein \\
\hline Os.32115.1.S1_at & 5.81 & 2.54 & LOC_Os01g71350 & Glucan endo-1,3-beta-glucosidase GII \\
\hline Os.27793.1.S1_at & 6.13 & 2.62 & LOC_Os02g14430 & Peroxidase 52 \\
\hline Os.405.1.S1_a_at & 6.22 & 2.64 & LOC_Os $12 \mathrm{~g} 37260$ & Lipoxygenase 2.1 \\
\hline Os.26511.1.S̄1_at & 6.24 & 2.64 & LOC_Os04g49370 & expressed protein \\
\hline Os.10255.1.s1_s_at & 6.28 & 2.65 & LOC_Os10g03850.3 & F-box domain containing protein \\
\hline Os.54545.1.s1_at & 6.36 & 2.67 & LOC_Os07g41060 & Dihydroflavonol-4-reductase \\
\hline Os.55259.1.S1_at & 6.39 & 2.68 & LOC_Os04g32480 & ZIM motif family protein \\
\hline Os.11164.1.S1_at & 6.51 & 2.70 & LOC_Os07g03279.1 & SCP-like extracellular protein \\
\hline Os.9660.1.S1_at & 6.64 & 2.73 & LOC_Os09g23300 & Integral membrane protein \\
\hline Os.51641.1.s1_at & 6.77 & 2.76 & LOC_Os07g03590 & Basic form of pathogenesis-related protein 1 \\
\hline Os.56018.1.S1_at & 7.24 & 2.86 & LOC_Os05g44060 & expressed protein \\
\hline Os.7935.1.S1_at & 7.28 & 2.86 & LOC_Os07g46852.1 & oxidoreductase, short chain dehydrogenase/reductase family protein \\
\hline Os.29987.2.s1_at & 7.29 & 2.87 & LOC_Os05g50610.1 & retrotransposon protein, putative, Ty3-gypsy subclass \\
\hline Os.10510.1.S1_at & 7.30 & 2.87 & LOC_Os03g03034.1 & Naringenin 3-dioxygenase \\
\hline Os.25606.1.S1_at & 7.32 & 2.87 & LOC_Os09g25060 & WRKY DNA binding domain containing protein \\
\hline Os.11812.1.S1_at & 7.60 & 2.93 & LOC_Os02g48770 & SAM dependent carboxyl methyltransferase family protein \\
\hline Os.51637.1.S1_x_at & 7.72 & 2.95 & LOC_Os07g31920.1 & transposon protein, putative, CACTA, En/Spm sub-class \\
\hline Os.17112.1.s1_at & 7.73 & 2.95 & LOC_Os03g18030 & Flavonol synthase \\
\hline Os.11552.1.S1_at & 7.79 & 2.96 & LOC_Os04g59150 & Peroxidase 12 \\
\hline Os.50342.1.S1 at & 7.93 & 2.99 & LOC Os04g52780 & Leucine-rich repeat receptor protein kinase EXS precursor \\
\hline Os.12713.1.S2_a_at & 8.74 & 3.13 & LOC_Os01g03320 & Bowman-Birk type bran trypsin inhibitor \\
\hline Os.159.1.S1_s_at & 9.62 & 3.27 & LOC_Os03g13210 & Peroxidase $\mathrm{N}$ \\
\hline Os.12952.1.S1_at & 10.96 & 3.45 & LOC_Os01g71860 & Glucan endo-1,3-beta-glucosidase GII \\
\hline Os.57103.1.S1_at & 11.33 & 3.50 & LOC_Os02g02930 & Terpene synthase family, metal binding domain containing protein \\
\hline Os.38638.1.S1_at & 11.39 & 3.51 & LOC_Os06g04990 & Early nodulin 93 \\
\hline Os.10908.1.s1_a_at & 12.14 & 3.60 & LOC_Os08g36910 & alpha-amylase isozyme 3D \\
\hline Os.55273.1.s1_at & 13.85 & 3.79 & LOC_Os04g34180.1 & hypothetical protein \\
\hline Os.54934.1.S1_at & 16.88 & 4.08 & LOC_Os05g37060 & Myb-like DNA-binding domain containing protein \\
\hline Os.31778.1.S1_x_at & 19.15 & 4.26 & LOC_Os01g61610 & oxidoreductase, $20 \mathrm{G}-\mathrm{Fe}$ oxygenase family protein \\
\hline Os.30000.1.S1_at & 19.35 & 4.27 & LOC_Os07g36560 & Transferase family protein \\
\hline Os.6863.1.S1_at & 32.02 & 5.00 & LOC_Os12g14440 & expressed protein \\
\hline
\end{tabular}




\section{Further Reading}

Caister Academic Press is a leading academic publisher of advanced texts in microbiology, molecular biology and medical research. Full details of all our publications at caister.com

- MALDI-TOF Mass Spectrometry in Microbiology Edited by: M Kostrzewa, S Schubert (2016) www.caister.com/malditof

- Aspergillus and Penicillium in the Post-genomic Era Edited by: RP Vries, IB Gelber, MR Andersen (2016) www.caister.com/aspergillus2

- The Bacteriocins: Current Knowledge and Future Prospects Edited by: RL Dorit, SM Roy, MA Riley (2016)

www.caister.com/bacteriocins

- Omics in Plant Disease Resistance Edited by: V Bhadauria (2016) www.caister.com/opd

- Acidophiles: Life in Extremely Acidic Environments Edited by: R Quatrini, DB Johnson (2016) www.caister.com/acidophiles

- Climate Change and Microbial Ecology: Current Research and Future Trend

Edited by: J Marxsen (2016)

www.caister.com/climate

- Biofilms in Bioremediation: Current Research and Emerging Technologies

Edited by: G Lear (2016)

www.caister.com/biorem

- Microalgae: Current Research and Applications Edited by: MN Tsaloglou (2016) www.caister.com/microalgae

- Gas Plasma Sterilization in Microbiology: Theory, Applications, Pitfalls and New Perspectives Edited by: H Shintani, A Sakudo (2016) www.caister.com/gasplasma

- Virus Evolution: Current Research and Future Directions Edited by: SC Weaver, M Denison, M Roossinck, et al. (2016) www.caister.com/virusevol

- Arboviruses: Molecular Biology, Evolution and Control Edited by: N Vasilakis, DJ Gubler (2016) www.caister.com/arbo

- Shigella: Molecular and Cellular Biology Edited by: WD Picking, WL Picking (2016) www.caister.com/shigella

-Aquatic Biofilms: Ecology, Water Quality and Wastewater Treatment

Edited by: AM Romaní, H Guasch, MD Balaguer (2016)

www.caister.com/aquaticbiofilms

- Alphaviruses: Current Biology

Edited by: S Mahalingam, L Herrero, B Herring (2016)

www.caister.com/alpha

- Thermophilic Microorganisms

Edited by: F Li (2015)

www.caister.com/thermophile
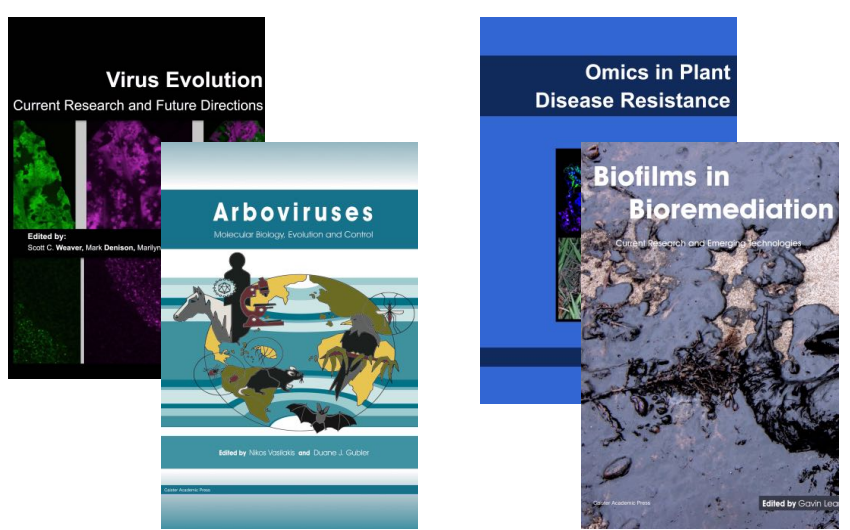
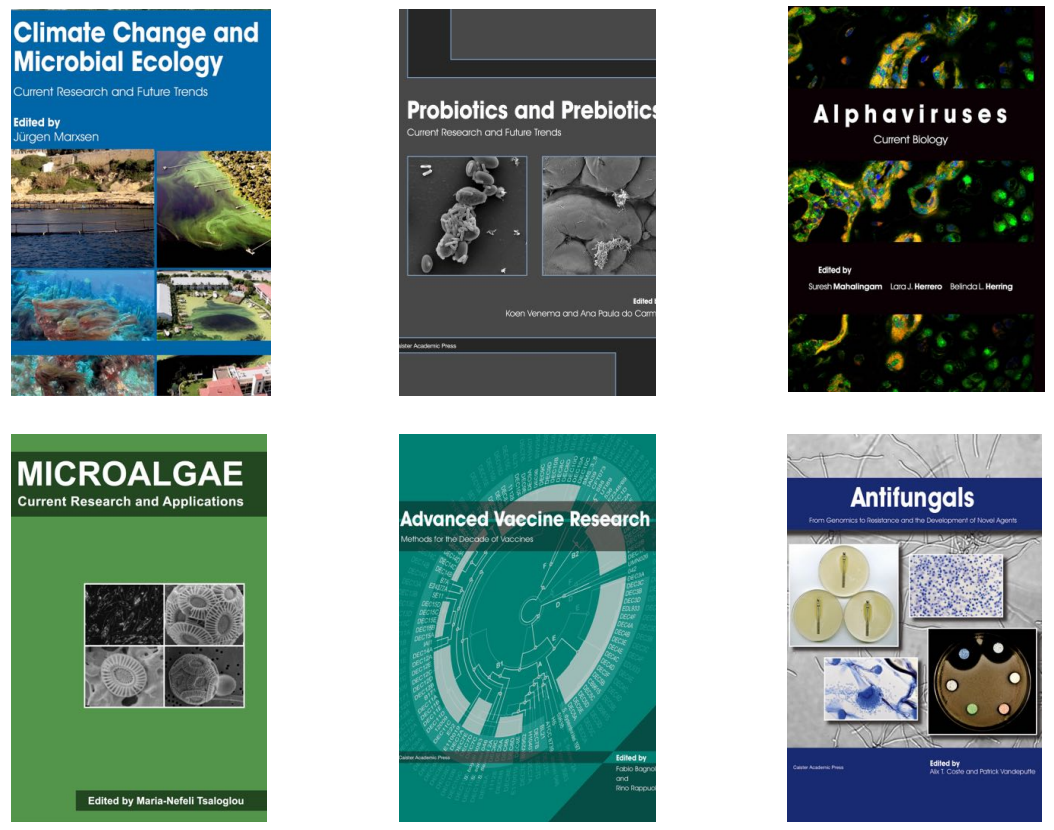

- Flow Cytometry in Microbiology: Technology and Applications Edited by: MG Wilkinson (2015) www.caister.com/flow

- Probiotics and Prebiotics: Current Research and Future Trends Edited by: K Venema, AP Carmo (2015) www.caister.com/probiotics

- Epigenetics: Current Research and Emerging Trends Edited by: BP Chadwick (2015) www.caister.com/epigenetics2015

- Corynebacterium glutamicum: From Systems Biology to Biotechnological Applications

Edited by: A Burkovski (2015)

www.caister.com/cory2

- Advanced Vaccine Research Methods for the Decade of Vaccines

Edited by: F Bagnoli, R Rappuoli (2015)

www.caister.com/vaccines

- Antifungals: From Genomics to Resistance and the Development of Novel Agents

Edited by: AT Coste, P Vandeputte (2015)

www.caister.com/antifungals

- Bacteria-Plant Interactions: Advanced Research and Future Trends Edited by: J Murillo, BA Vinatzer, RW Jackson, et al. (2015) www.caister.com/bacteria-plant

\section{- Aeromonas}

Edited by: J Graf (2015)

www.caister.com/aeromonas

- Antibiotics: Current Innovations and Future Trends

Edited by: S Sánchez, AL Demain (2015)

www.caister.com/antibiotics

- Leishmania: Current Biology and Contro Edited by: S Adak, R Datta (2015) www.caister.com/leish2

- Acanthamoeba: Biology and Pathogenesis (2nd edition) Author: NA Khan (2015)

www.caister.com/acanthamoeba2

- Microarrays: Current Technology, Innovations and Applications Edited by: Z He (2014)

www.caister.com/microarrays2

- Metagenomics of the Microbial Nitrogen Cycle: Theory, Methods and Applications

Edited by: D Marco (2014)

www.caister.com/n2 